\title{
Direction and Competitiveness of Cotton Export under WTO Regime
}

\author{
J.A. Lamtule ${ }^{1 *}$, P.P. Sawant ${ }^{1}$ and R.G. Deshmukh ${ }^{2}$ \\ ${ }^{1}$ College of ABM, Gunjalwadi Pathar, Sangamner, Ahmednagar, India \\ ${ }^{2}$ Department of Agriculture Economics and Statistics, Dr. P. D. K. V., Akola, (M.S.), India \\ *Corresponding author
}

\section{A B S T R A C T}

\section{Keywords}

Pre- and Post-WTO,

Competitiveness,

Cotton

Article Info

Accepted:

10 December 2017

Available Online:

10 January 2018
The present study attempts to examine the direction and competitiveness of cotton export during pre- and post-WTO period. The time series data on export and import were collected for a period from $1977-78$ to $2012-13$. The study period was divided into preWTO period (1977-78 to 1994-95) and post-WTO period (1995-96 to 2012-13). The findings of the study reveal that during pre-WTO period Bangladesh, Portugal, Singapore, Spain, Sri Lanka, Switzerland, UAE, UK, and USA were highly unstable importers of Indian cotton. It is observed that during post-WTO period Bangladesh, Indonesia, Nepal, Portugal, Republic of Korea, Singapore, Spain, Sri Lanka, Switzerland and UAE were highly unstable importers of Indian cotton. While China and Japan were the most stable importers of Indian cotton during post-WTO period. The results of the NPC values for both the pre-WTO and post-WTO period indicated that the coefficients were less than one for all the years. It indicate that there was a more scope for export of cotton i.e. cotton was dis-protected in India. The average NPC value for pre-WTO period (0.34) and post-WTO period $(0.38)$ indicated that the unit price of the Indian cotton in the domestic market was not much competitive in the international market.

\section{Introduction}

Cotton is one of the most important textile fiber in the world, accounting for 35 per cent of the world fiber use. Among the cotton growing countries, India has the largest area under cotton production followed by China, United States and Pakistan. India accounts for about 34 per cent of the global cotton area and contributes to 28 per cent of the global cotton production. China is the largest producer of cotton followed by India and United states. Though China (5.5 million hectares) has just more than half of India's area (12.2 million hectares), it produces twice the cotton compared to India. Among the six major cotton growing countries, Brazil (1353 kg/ha) hold highest productivity level followed by China (1346 kg/ha), USA (886 kg/ha), Uzbekistan (698 kg/ha), Pakistan (769 kg/ha) and India (491 kg/ha). China is the largest consumer followed by India and Pakistan. United States is the largest exporter followed by India and Uzbekistan. While the major importers of cotton are China, Turkey, Bangladesh and Pakistan. Though the share of agricultural exports has been declining over the years (18-20 per cent in 1990), there are 
certain commodities whose exports are increasing and one such commodity is cotton. Cotton is the only crop in which India enjoys genuine export surplus after meeting our domestic needs and the raw material availability has not been an issue for industries in the last few years. A Cotton export is one of the major issues in the WTO as it is a major crop of the developing countries who suffered the loss of price fall due to subsidies.

Against this back ground the present study is undertaken to estimate the direction and competitiveness of cotton export during preand post -WTO periods.

\section{Hypothesis}

The Indian cotton exports have increased in the post WTO period.

The Indian cotton is competitive in international market.

\section{Materials and Methods}

The study is based on the secondary data collected from various published sources. The time series data regarding export and import of cotton was collected from 1977-78 to 201213 and this period was divided into pre-WTO period (1977-78 to 1994-95) and post-WTO period (1995-96 to 2012-13). The data on world production of cotton and exchange rate were obtained from the FAO publications and the publications of the Directorate General of Commerce, Intelligence and Statistics (DGCIS), Kolkata.

The trade direction of Indian cotton exports were analyzed by using the first order Markov chain approach (Jayesh, 2001). Central to Markov chain analysis is the estimation of the transitional probability matrix $\mathrm{P}$. The elements $\mathrm{P}_{\mathrm{ij}}$ of the matrix $\mathrm{P}$ indicates the probability that export will switch from country $i$ to country $j$ with the passage of time. The diagonal elements of the matrix measure the probability that the export share of a country will be retained. Hence, an examination of the diagonal elements indicates the loyalty of an importing country to a particular country's exports.

The export competitiveness of cotton was analyzed by using nominal protection coefficient. The nominal protection coefficient (NPC) is defined as the ratio of the domestic price to the world reference price of the commodity under consideration. Symbolically,

$$
N P C=\frac{P d}{\operatorname{Pr}}
$$

Where,

$\mathrm{NPC}=$ nominal protection coefficient

$\mathrm{Pd}=$ domestic price of the cotton

$\operatorname{Pr}=$ world reference price of the cotton i.e., what the farmer would have received in case of free trade.

If the NPC is greater than one, then the commodity is protected (no scope for export), compared to the situation what would prevail under free trade and if it is less than one the commodity is dis-protected (scope for export).

\section{Results and Discussion}

The results obtained from the present study have been discussed in detail as under:

\section{Direction of cotton exports from India}

Indian cotton is exported to about thirty one countries in the world. For the purpose of studying the directions of exports, twenty 
major countries were selected based on the maximum amount of export to these countries and rests of the countries were grouped as others. The destination wise export of Indian cotton is given in Table 1.

The transitional probability matrices are presented in Table 2 and 3. The results indicated the changes in the direction of trade of Indian cotton in both periods which helped in describing the changing direction during the post-WTO period over the pre-WTO period.

It is observed from Table 2 that during preWTO period Bangladesh, Portugal, Singapore, Spain, Sri Lanka, Switzerland, UAE, UK, and USA were highly unstable importers of Indian cotton and could not retain their previous year's share of cotton export. The previous period's share of Portugal, Singapore, UK and USA were shifted to Spain. While China, Turkey, Indonesia, Japan and Thailand were the most stable importers of Indian cotton during pre-WTO period.

The sum of diversified and retained export for each country was given below in the form of transitional probabilities. The picture of export indicates that there was 5.21 per cent increase in export to China during pre-WTO period. Similarly highest increase of 89.63 per cent in export to Spain followed by 45.91 per cent to Indonesia, 42.26 per cent to UK, 36.44 per cent to Mauritius, 16.99 per cent to Philippines, 16.38 per cent to Bangladesh and 12.52 per cent in export to Japan were observed. At the same time there was 74.66 per cent reduction in export to Sri Lanka, 72.97 per cent to USA, 70.17 per cent to UK, 67.14 per cent to Singapore, 54.02 per cent to Portugal, and 18.92 per cent reduction in export to Vietnam during the pre-WTO period.

The trade pattern and direction of cotton exports during post-WTO period is given in the Table 3. The transitional probability matrix gives a broad indication of the changes in the direction of trade of Indian cotton exports over a period of 18 years. It is observed from Table 3 that during post-WTO period Bangladesh, Indonesia, Nepal, Portugal, Republic of Korea, Singapore, Spain, Sri Lanka, Switzerland and UAE were highly unstable importers of Indian cotton and could not retain their previous year's share of cotton export.

The previous period's share of Bangladesh, Indonesia, Portugal and Singapore were shifted to China. While China and Japan were the most stable importers of Indian cotton during post-WTO period.

The sum of diversified and retained export for each country was given below in the form of transitional probabilities. The picture of export indicates that there was 223.06 per cent increase in export to Spain during post-WTO period followed by 146.11 per cent to Japan, 42.72 per cent to UK, 33.25 per cent to USA, 28.04 per cent to Sri Lanka, 23.41 per cent to Thailand and 16.88 per cent in export to others were observed.

At the same time there was 60.81 per cent reduction in export to Nepal, 56.97 per cent to UAE, 49.36 per cent to Switzerland, 44.61 per cent to Korea, 32.21 per cent to Mauritius, and 25 per cent reduction in export to Portugal during the post-WTO period.

\section{Direction of cotton imports by India}

India imports cotton from about seventy five countries in the world. For the purpose of studying the directions of imports, twenty major countries were selected based on the maximum amount of import from these countries and rests of the countries were grouped as others. The destination wise import of cotton by India is given in Table 4 . 
Table.1 Export of Indian cotton to different Destinations

\begin{tabular}{|c|c|c|c|c|c|}
\hline \multirow[t]{2}{*}{ Sr. No. } & \multirow[t]{2}{*}{ Country } & \multicolumn{2}{|c|}{ Pre-WTO Period } & \multicolumn{2}{|c|}{ Post-WTO Period } \\
\hline & & $\begin{array}{c}\text { Average Export } \\
\text { (Tons) }\end{array}$ & Share (\%) & $\begin{array}{c}\text { Average Export } \\
\text { (Tons) }\end{array}$ & Share $(\%)$ \\
\hline 1 & China & 27257.22 & 31.89 & 284654.83 & 55.54 \\
\hline 2 & Bangladesh & 1714.72 & 2.01 & 57935.44 & 11.30 \\
\hline 3 & Indonesia & 3701.11 & 4.33 & 25934.72 & 5.06 \\
\hline 4 & Thailand & 5942.83 & 6.95 & 14063.06 & 2.74 \\
\hline 5 & Vietnam & 1835.94 & 2.15 & 14764.11 & 2.88 \\
\hline 6 & Turkey & 4926.56 & 5.76 & 13503.56 & 2.63 \\
\hline 7 & Japan & 13831.78 & 16.18 & 5880.83 & 1.15 \\
\hline 8 & Malaysia & 1651.61 & 1.93 & 5375.89 & 1.05 \\
\hline 9 & Mauritius & 206.06 & 0.24 & 3154.39 & 0.62 \\
\hline$\overline{10}$ & Nepal & 208.00 & 0.24 & 962.44 & 0.19 \\
\hline 11 & Philippines & 513.67 & 0.60 & 1098.44 & 0.21 \\
\hline$\overline{12}$ & Portugal & 645.33 & 0.75 & 471.22 & 0.09 \\
\hline$\overline{13}$ & Republic of Korea & 2868.89 & 3.36 & 2114.00 & 0.41 \\
\hline$\overline{14}$ & Singapore & 3583.61 & 4.19 & 2109.67 & 0.41 \\
\hline 15 & Spain & 3479.67 & 4.07 & 39.28 & 0.01 \\
\hline 16 & Sri Lanka & 2579.28 & 3.02 & 267.61 & 0.05 \\
\hline 17 & Switzerland & 198.33 & 0.23 & 135.94 & 0.03 \\
\hline$\overline{18}$ & UAE & 39.28 & 0.05 & 1135.17 & 0.22 \\
\hline 19 & UK & 1472.33 & 1.72 & 639.22 & 0.12 \\
\hline 20 & USA & 1598.94 & 1.87 & 990.22 & 0.19 \\
\hline \multirow[t]{2}{*}{21} & Others & 7224.22 & 8.45 & 77313.89 & 15.08 \\
\hline & Total & 85479.39 & 100 & 512543.9444 & 100 \\
\hline
\end{tabular}

Table.4 Import of cotton by India from different Destinations

\begin{tabular}{|c|c|c|c|c|c|c|}
\hline \multirow[t]{2}{*}{ Sr. No. } & \multicolumn{3}{|l|}{ Pre-WTO Period } & \multicolumn{3}{|l|}{ Post-WTO Period } \\
\hline & Country & Average Import (Tons) & Share $(\%)$ & Country & Average Import (Tons) & Share (\%) \\
\hline 1 & Australia & 2269.78 & 11.53 & Australia & 8180.50 & 6.23 \\
\hline 2 & Benin & 1157.39 & 5.88 & Benin & 6043.06 & 4.60 \\
\hline 3 & Burkina Faso & 544.39 & 2.77 & Burkina Faso & 4439.72 & 3.38 \\
\hline 4 & Cote d'lvoire & 810.67 & 4.12 & Cote d'lvoire & 5111.39 & 3.89 \\
\hline 5 & Egypt & 1819.78 & 9.25 & Egypt & 16407.83 & 12.50 \\
\hline 6 & Mali & 559.44 & 2.84 & Mali & 4413.72 & 3.36 \\
\hline 7 & Pakistan & 5407.06 & 27.48 & Sudan & 3465.17 & 2.64 \\
\hline 8 & Sudan & 2210.94 & 11.23 & Tanzania & 5955.89 & 4.54 \\
\hline 9 & Tanzania & 283.83 & 1.44 & USA & 35381.94 & 26.95 \\
\hline 10 & USA & 2151.22 & 10.93 & Uzbekistan & 4768.17 & 3.63 \\
\hline 11 & Uzbekistan & 211.06 & 1.07 & Argentina & 945.22 & 0.72 \\
\hline 12 & Cameroon & 76.89 & 0.39 & Cameroon & 2222.78 & 1.69 \\
\hline 13 & China & 171.78 & 0.87 & China & 1722.39 & 1.31 \\
\hline 15 & Malaysia & 13.11 & 0.07 & Israel & 887.33 & 0.68 \\
\hline 16 & Russian Federation & 110.17 & 0.56 & Malaysia & 1032.50 & 0.79 \\
\hline 17 & Switzerland & 191.44 & 0.97 & Nigeria & 1276.44 & 0.97 \\
\hline 18 & Turkey & 223.22 & 1.13 & Russian Federation & 1610.39 & 1.23 \\
\hline 19 & Turkmenistan & 133.78 & 0.68 & South Africa & 2085.61 & 1.59 \\
\hline 20 & Austria & 4.39 & 0.02 & Switzerland & 802.78 & 0.61 \\
\hline 21 & Other & 1244.44 & 6.32 & Others & 21394.67 & 16.29 \\
\hline & Total & 19679.22 & 100 & Total & 131298.83 & 100 \\
\hline
\end{tabular}


Int.J.Curr.Microbiol.App.Sci (2018) 7(1): 834-844

Table.7 Nominal protection coefficient of cotton

\begin{tabular}{|c|c|c|c|c|c|c|c|}
\hline \multicolumn{4}{|l|}{ Pre-WTO } & \multicolumn{4}{|c|}{ Post-WTO } \\
\hline Year & $\begin{array}{l}\text { Domestic } \\
\text { Price } \\
\text { (Rs/qtl) }\end{array}$ & $\begin{array}{l}\text { International } \\
\text { Price (Rs/qtl) }\end{array}$ & NPC & Year & $\begin{array}{l}\text { Domestic } \\
\text { Price (Rs/qtl) }\end{array}$ & $\begin{array}{l}\text { International } \\
\text { Price (Rs/qtl) }\end{array}$ & NPC \\
\hline 77-78 & 380 & 801 & 0.47 & $95-96$ & 1350 & 4133 & 0.33 \\
\hline 78-79 & 410 & 1446 & 0.28 & $96-97$ & 1380 & 6768 & 0.20 \\
\hline $79-80$ & 445 & 1548 & 0.29 & $97-98$ & 1530 & 5887 & 0.26 \\
\hline $80-81$ & 475 & 1150 & 0.41 & 98-99 & 1650 & 5843 & 0.28 \\
\hline 81-82 & 480 & 1324 & 0.36 & $99-2000$ & 1775 & 5102 & 0.35 \\
\hline $82-83$ & 515 & 1535 & 0.34 & $2000-01$ & 1825 & 5122 & 0.36 \\
\hline 83-84 & 527 & 1482 & 0.36 & 2001-02 & 1875 & 5095 & 0.37 \\
\hline $84-85$ & 535 & 1503 & 0.36 & 2002-03 & 1875 & 5214 & 0.36 \\
\hline $85-86$ & 535 & 1997 & 0.27 & 2003-04 & 1925 & 4131 & 0.47 \\
\hline $86-87$ & 540 & 1522 & 0.35 & 2004-05 & 1960 & 4636 & 0.42 \\
\hline 87-88 & 550 & 1153 & 0.48 & $2005-06$ & 1980 & 4552 & 0.44 \\
\hline 88-89 & 600 & 1465 & 0.41 & 2006-07 & 1990 & 4845 & 0.41 \\
\hline $89-90$ & 690 & 3148 & 0.22 & 2007-08 & 2030 & 4817 & 0.42 \\
\hline $90-91$ & 750 & 3966 & 0.19 & 2008-09 & 3000 & 6015 & 0.50 \\
\hline $91-92$ & 840 & 3037 & 0.28 & 2009-10 & 3000 & 7070 & 0.42 \\
\hline $92-93$ & 950 & 2008 & 0.47 & 2010-11 & 3000 & 6681 & 0.45 \\
\hline 93-94 & 1050 & 3413 & 0.31 & 2011-12 & 3300 & 8861 & 0.37 \\
\hline $94-95$ & 1200 & 3380 & 0.36 & 2012-13 & 3900 & 9697 & 0.40 \\
\hline Average & 637.33 & 1993.23 & 0.34 & Average & 2185.83 & 5803.89 & 0.38 \\
\hline
\end{tabular}


Table.2 Transitional probability matrix of cotton exports in the pre-WTO period (1977-78 to 1994-95)

\begin{tabular}{|c|c|c|c|c|c|c|c|c|c|c|c|c|c|c|c|c|c|c|c|c|c|c|}
\hline & China & Bangladesh & Indonesia & Thailand & Viet Nam & Turkey & Japan & Malaysia & Mauritius & Nepal & Philippines & Portugal & $\begin{array}{c}\text { R. of } \\
\text { Korea }\end{array}$ & Singapore & Spain & $\begin{array}{c}\text { Sri } \\
\text { Lanka }\end{array}$ & Switzerland & UAE & UK & USA & Others & $\begin{array}{c}\text { Average } \\
\text { Export }\end{array}$ \\
\hline China & \begin{tabular}{|c|}
0.7518 \\
$(20492.18)$ \\
\end{tabular} & $\begin{array}{c}0.0328 \\
(894.48) \\
\end{array}$ & & $\begin{array}{c}0.0102 \\
(278.36) \\
\end{array}$ & $\begin{array}{l}0.0000 \\
(0.00) \\
\end{array}$ & $\begin{array}{c}0.0189 \\
(514.79) \\
\end{array}$ & \begin{tabular}{|c|}
0.1827 \\
$(4978.89)$ \\
\end{tabular} & & & $\begin{array}{c}0.0000 \\
(0.00) \\
\end{array}$ & & & & & $\begin{array}{l}0.0000 \\
(0.00) \\
\end{array}$ & & & $\begin{array}{l}0.0002 \\
(5.26) \\
\end{array}$ & \begin{tabular}{|c|}
0.0000 \\
$(0.00)$ \\
\end{tabular} & & & 27257.22 \\
\hline Bangladesh & \begin{tabular}{|c|}
0.6180 \\
$(1059.64)$ \\
\end{tabular} & $\begin{array}{c}.0000 \\
(0.00)\end{array}$ & $\begin{array}{c}0.3347 \\
(573.95) \\
\end{array}$ & $\begin{array}{l}0.0000 \\
(0.00)\end{array}$ & $\begin{array}{l}0.0000 \\
(0.00)\end{array}$ & $\begin{array}{c}0.0000 \\
(0.00) \\
\end{array}$ & $\begin{array}{c}0.0000 \\
(0.00) \\
\end{array}$ & $\begin{array}{l}0.0443 \\
(75.94) \\
\end{array}$ & $\begin{array}{l}0.0000 \\
(0.00)\end{array}$ & $\begin{array}{c}0.0000 \\
(0.00) \\
\end{array}$ & $\begin{array}{l}0.0000 \\
(0.00)\end{array}$ & $\begin{array}{l}0.0000 \\
(0.00)\end{array}$ & $\begin{array}{l}0.0000 \\
(0.00)\end{array}$ & $\begin{array}{l}0.0000 \\
(0.00)\end{array}$ & $\begin{array}{l}0.0000 \\
(0.00)\end{array}$ & $\begin{array}{l}0.0000 \\
(0.00)\end{array}$ & $\begin{array}{l}0.0030 \\
(5.19) \\
\end{array}$ & $\begin{array}{l}.0000 \\
(0.00)\end{array}$ & \begin{tabular}{|c|}
0.0000 \\
$(0.00)$ \\
\end{tabular} & $\begin{array}{c}.0000 \\
(0.00)\end{array}$ & $\begin{array}{l}0.0000 \\
(0.00)\end{array}$ & 1714.72 \\
\hline Indonesia & $\begin{array}{c}0.0000 \\
(0.00) \\
\end{array}$ & $\begin{array}{l}0.0000 \\
(0.00)\end{array}$ & \begin{tabular}{c|}
0.5803 \\
$(2147.90)$ \\
\end{tabular} & \begin{tabular}{|c|}
0.3693 \\
$(1366.68)$ \\
\end{tabular} & $\begin{array}{l}0.0000 \\
(0.00) \\
\end{array}$ & $\begin{array}{l}0.0000 \\
(0.00) \\
\end{array}$ & $\begin{array}{l}0.0000 \\
(0.00) \\
\end{array}$ & $\begin{array}{c}0.0298 \\
(110.24) \\
\end{array}$ & $\begin{array}{l}0.0000 \\
(0.00) \\
\end{array}$ & \begin{tabular}{|l|}
0.0044 \\
$(16.27)$ \\
\end{tabular} & $\begin{array}{l}0.0092 \\
(34.23) \\
\end{array}$ & $\begin{array}{l}0.0000 \\
(0.00)\end{array}$ & $\begin{array}{c}0.0000 \\
(0.00)\end{array}$ & $\begin{array}{l}0.0000 \\
(0.00) \\
\end{array}$ & $\begin{array}{l}0.0000 \\
(0.00)\end{array}$ & \begin{tabular}{|c|}
0.0000 \\
$(0.00)$ \\
\end{tabular} & $\begin{array}{l}0.0000 \\
(0.00)\end{array}$ & $\begin{array}{l}0.0000 \\
(0.00)\end{array}$ & $\begin{array}{l}0.0000 \\
(0.00) \\
\end{array}$ & $\begin{array}{l}0.0070 \\
(25.79) \\
\end{array}$ & $\begin{array}{l}0.0000 \\
(0.00) \\
\end{array}$ & 3701.11 \\
\hline Thailand & $\begin{array}{c}0.0000 \\
(0.00) \\
\end{array}$ & $\begin{array}{c}0.1050 \\
(623.84) \\
\end{array}$ & $\begin{array}{c}0.2536 \\
(1506.94) \\
\end{array}$ & \begin{tabular}{|c|}
$\mathbf{0 . 5 0 7 3}$ \\
$(\mathbf{3 0 1 4 . 5 3 )}$ \\
\end{tabular} & $\begin{array}{l}0.0000 \\
(0.00) \\
\end{array}$ & $\begin{array}{l}0.0000 \\
(0.00) \\
\end{array}$ & $\begin{array}{l}0.0000 \\
(0.00) \\
\end{array}$ & $\begin{array}{l}0.0100 \\
(59.38) \\
\end{array}$ & $\begin{array}{l}0.0000 \\
(0.00) \\
\end{array}$ & \begin{tabular}{|l|}
0.0070 \\
$(41.71)$ \\
\end{tabular} & $\begin{array}{c}0.0595 \\
(353.89) \\
\end{array}$ & $\begin{array}{l}0.0052 \\
(31.00) \\
\end{array}$ & $\begin{array}{l}0.0000 \\
(0.00)\end{array}$ & $\begin{array}{l}0.0015 \\
(0.00) \\
\end{array}$ & $\begin{array}{l}0.0000 \\
(0.00) \\
\end{array}$ & \begin{tabular}{|c|}
0.0000 \\
$(0.00)$ \\
\end{tabular} & $\begin{array}{l}0.0082 \\
(48.57) \\
\end{array}$ & $\begin{array}{l}0.0014 \\
(8.38) \\
\end{array}$ & $\begin{array}{l}0.0000 \\
(0.00) \\
\end{array}$ & $\begin{array}{c}0.0000 \\
(0.00)\end{array}$ & $\begin{array}{c}0.0413 \\
(245.66) \\
\end{array}$ & 5942.83 \\
\hline Viet Nam & $\begin{array}{c}0.6764 \\
(1241.74) \\
\end{array}$ & $\begin{array}{c}0.0000 \\
(0.00)\end{array}$ & $\begin{array}{l}0.0000 \\
(0.00) \\
\end{array}$ & $\begin{array}{c}0.0000 \\
(0.00) \\
\end{array}$ & \begin{tabular}{|c|}
0.2894 \\
$(531.38)$ \\
\end{tabular} & $\begin{array}{l}0.0000 \\
(0.00) \\
\end{array}$ & $\begin{array}{l}0.0000 \\
(0.00)\end{array}$ & $\begin{array}{l}0.0342 \\
(62.82) \\
\end{array}$ & & $\begin{array}{c}0.0000 \\
(0.00) \\
\end{array}$ & & & $\begin{array}{c}0.0000 \\
(0.00)\end{array}$ & & & $\begin{array}{c}0.0000 \\
(0.00) \\
\end{array}$ & & $\begin{array}{l}0.0000 \\
(0.00)\end{array}$ & $\begin{array}{l}0.0000 \\
(0.00) \\
\end{array}$ & & & 1835.94 \\
\hline Turkey & $\begin{array}{l}0.0000 \\
(0.00)\end{array}$ & $\begin{array}{l}0.0000 \\
(0.00)\end{array}$ & & $\begin{array}{l}0.0000 \\
(0.00)\end{array}$ & & \begin{tabular}{|c|}
$\begin{array}{c}0.5984 \\
(2948.26)\end{array}$ \\
\end{tabular} & $\begin{array}{c}0.1805 \\
(889.25) \\
\end{array}$ & \begin{tabular}{|c|}
0.2159 \\
$(1063.48)$
\end{tabular} & & & & & & & & & & & $\begin{array}{l}0.0000 \\
(0.00)\end{array}$ & & & 4926.56 \\
\hline Japan & $\begin{array}{c}0.2395 \\
(3312.71)\end{array}$ & $\begin{array}{c}0.0297 \\
(410.80)\end{array}$ & & & & & \begin{tabular}{|c|}
$\begin{array}{c}0.5513 \\
(7624.77)\end{array}$ \\
\end{tabular} & & & & & & & & & & & & \begin{tabular}{|l|}
0.0011 \\
$(15.21)$
\end{tabular} & & & 13831.78 \\
\hline Malaysia & $\begin{array}{c}0.1523 \\
(251.59)\end{array}$ & & & $\begin{array}{l}0.0000 \\
(0.00)\end{array}$ & \begin{tabular}{|c|}
0.1610 \\
$(265.95)$ \\
\end{tabular} & \begin{tabular}{|c|}
0.4891 \\
$(807.87)$ \\
\end{tabular} & & & & & & & & & & & & & \begin{tabular}{|c|}
0.0000 \\
$(0.00)$
\end{tabular} & & & 1651.61 \\
\hline Mauritius & $\begin{array}{l}0.4562 \\
(94.00) \\
\end{array}$ & $\begin{array}{l}0.1321 \\
(27.22) \\
\end{array}$ & $\begin{array}{l}0.1243 \\
(25.61) \\
\end{array}$ & $\begin{array}{l}0.0000 \\
(0.00)\end{array}$ & $\begin{array}{l}0.0000 \\
(0.00) \\
\end{array}$ & $\begin{array}{l}.0000 \\
(0.00) \\
\end{array}$ & $\begin{array}{l}0.0000 \\
(0.00)\end{array}$ & $\begin{array}{c}0.0000 \\
(0.00)\end{array}$ & & $\begin{array}{c}0.0000 \\
(0.00) \\
\end{array}$ & $\begin{array}{l}0.0000 \\
(0.00)\end{array}$ & $\begin{array}{l}0.0000 \\
(0.00)\end{array}$ & $\begin{array}{l}0.0000 \\
(0.00)\end{array}$ & $\begin{array}{l}0.0000 \\
(0.00)\end{array}$ & $\begin{array}{l}0.0000 \\
(0.00)\end{array}$ & \begin{tabular}{|c|}
0.0000 \\
$(0.00)$ \\
\end{tabular} & & $\begin{array}{l}0.0000 \\
(0.00)\end{array}$ & $\begin{array}{l}0.0000 \\
(0.00) \\
\end{array}$ & & & 206.06 \\
\hline Nepal & $\begin{array}{c}0.6690 \\
(139.16) \\
\end{array}$ & $\begin{array}{c}0.0000 \\
(0.00) \\
\end{array}$ & $\begin{array}{l}0.0000 \\
(0.00)\end{array}$ & $\begin{array}{l}0.0000 \\
(0.00)\end{array}$ & $\begin{array}{l}0.0000 \\
(0.00)\end{array}$ & $\begin{array}{l}0.0000 \\
(0.00)\end{array}$ & $\begin{array}{l}0.0000 \\
(0.00) \\
\end{array}$ & $\begin{array}{l}0.0000 \\
(0.00)\end{array}$ & $\begin{array}{l}0.0000 \\
(0.00)\end{array}$ & \begin{tabular}{|l|}
0.3310 \\
$(68.84)$ \\
\end{tabular} & $\begin{array}{c}0.0000 \\
(0.00)\end{array}$ & $\begin{array}{c}0.0000 \\
(0.00)\end{array}$ & $\begin{array}{c}0.0000 \\
(0.00)\end{array}$ & $\begin{array}{l}0.0000 \\
(0.00) \\
\end{array}$ & $\begin{array}{l}0.0000 \\
(0.00)\end{array}$ & \begin{tabular}{|c|}
0.0000 \\
$(0.00)$ \\
\end{tabular} & $\begin{array}{l}0.0000 \\
(0.00) \\
\end{array}$ & $\begin{array}{c}0.0000 \\
(0.00)\end{array}$ & $\begin{array}{l}0.0000 \\
(0.00) \\
\end{array}$ & $\begin{array}{c}0.0000 \\
(0.00) \\
\end{array}$ & $\begin{array}{l}0.00 \\
(0.0\end{array}$ & 208.00 \\
\hline Philippines & $\begin{array}{c}0.7234 \\
(371.59) \\
\end{array}$ & $\begin{array}{c}0.0000 \\
(0.00) \\
\end{array}$ & $\begin{array}{c}0.0000 \\
(0.00) \\
\end{array}$ & $\begin{array}{c}0.0000 \\
(0.00) \\
\end{array}$ & $\begin{array}{l}0.0000 \\
(0.00) \\
\end{array}$ & $\begin{array}{l}0.0000 \\
(0.00) \\
\end{array}$ & $\begin{array}{l}0.0000 \\
(0.00) \\
\end{array}$ & $\begin{array}{l}0.0000 \\
(0.00) \\
\end{array}$ & $\begin{array}{l}0.0000 \\
(0.00) \\
\end{array}$ & \begin{tabular}{|l|}
0.0000 \\
$(0.00)$ \\
\end{tabular} & $\begin{array}{l}\mathbf{0 . 1 3 6 7} \\
(\mathbf{7 0 . 2 2}) \\
\end{array}$ & $\begin{array}{l}0.0000 \\
(0.00)\end{array}$ & $\begin{array}{l}0.0000 \\
(0.00)\end{array}$ & & \begin{tabular}{|c|}
0.0000 \\
$(0.00)$ \\
\end{tabular} & \begin{tabular}{|c|}
0.0000 \\
$(0.00)$ \\
\end{tabular} & & $\begin{array}{l}0.0000 \\
(0.00)\end{array}$ & $\begin{array}{l}0.0000 \\
(0.00)\end{array}$ & & & 513.67 \\
\hline Portugal & $\begin{array}{c}0.0000 \\
(0.00) \\
\end{array}$ & $\begin{array}{c}0.0000 \\
(0.00)\end{array}$ & $\begin{array}{c}0.0000 \\
(0.00)\end{array}$ & $\begin{array}{l}0.0000 \\
(0.00) \\
\end{array}$ & $\begin{array}{c}0.0000 \\
(0.00) \\
\end{array}$ & $\begin{array}{l}0.0000 \\
(0.00) \\
\end{array}$ & $\begin{array}{l}0.0000 \\
(0.00) \\
\end{array}$ & $\begin{array}{c}0.0000 \\
(0.00) \\
\end{array}$ & $\begin{array}{c}0.0000 \\
(0.00) \\
\end{array}$ & \begin{tabular}{|l|}
0.0259 \\
$(16.70)$ \\
\end{tabular} & & & $\begin{array}{c}0.0000 \\
(0.00)\end{array}$ & & \begin{tabular}{|c|}
0.9606 \\
$(619.89)$ \\
\end{tabular} & \begin{tabular}{|c|}
0.0000 \\
$(0.00)$ \\
\end{tabular} & & $\begin{array}{l}0.0000 \\
(0.00)\end{array}$ & $\begin{array}{l}0.0000 \\
(0.00) \\
\end{array}$ & & & 645.33 \\
\hline $\begin{array}{l}\text { Republic of } \\
\text { Korea }\end{array}$ & $\begin{array}{c}0.3661 \\
(1050.30) \\
\end{array}$ & $\begin{array}{c}0.0000 \\
(0.00)\end{array}$ & $\begin{array}{c}0.0590 \\
(169.21)\end{array}$ & $\begin{array}{l}0.0000 \\
(0.00)\end{array}$ & \begin{tabular}{|c|}
0.2087 \\
$(598.73)$ \\
\end{tabular} & $\begin{array}{l}0.0000 \\
(0.00)\end{array}$ & & & & & & & $\begin{array}{c}0.2223 \\
(637.79)\end{array}$ & & \begin{tabular}{|c|}
0.0000 \\
$(0.00)$
\end{tabular} & \begin{tabular}{|c|}
0.0000 \\
$(0.00)$
\end{tabular} & & $\begin{array}{l}0.0000 \\
(0.00)\end{array}$ & $\begin{array}{l}0.0000 \\
(0.00)\end{array}$ & $\begin{array}{l}0.0000 \\
(0.00)\end{array}$ & & 2868.89 \\
\hline Singapore & $\begin{array}{l}0.0000 \\
(0.00)\end{array}$ & & & & & & & & & & & & & & $\begin{array}{c}1.0000 \\
(3583.61)\end{array}$ & & & $\begin{array}{c}0.0000 \\
(0.00)\end{array}$ & $\begin{array}{c}0.0000 \\
(0.00)\end{array}$ & & & 3583.61 \\
\hline Spain & $\begin{array}{c}0.1910 \\
(664.75)\end{array}$ & & & $\begin{array}{c}0.2236 \\
(777.97)\end{array}$ & & $\begin{array}{l}0.0000 \\
(0.00)\end{array}$ & $\begin{array}{r}0.39 \\
(1387\end{array}$ & & & & & & & & & & & & $\begin{array}{l}0.0000 \\
(0.00)\end{array}$ & & & 3479.67 \\
\hline Sri Lanka & $\begin{array}{l}0.0000 \\
(0.00)\end{array}$ & & & $\begin{array}{l}0.0000 \\
(0.00)\end{array}$ & $\begin{array}{l}0.0000 \\
(0.00)\end{array}$ & $\begin{array}{l}0.0000 \\
(0.00)\end{array}$ & $\begin{array}{c}0.1889 \\
(487.31)\end{array}$ & $\begin{array}{l}0.0000 \\
(0.00)\end{array}$ & $\begin{array}{l}0.0000 \\
(0.00)\end{array}$ & $\begin{array}{c}0.0000 \\
(0.00)\end{array}$ & $\begin{array}{l}0.0000 \\
(0.00)\end{array}$ & $\begin{array}{l}0.0000 \\
(0.00)\end{array}$ & $\begin{array}{c}0.0540 \\
(139.20)\end{array}$ & $\begin{array}{l}0.0000 \\
(0.00)\end{array}$ & $\begin{array}{l}0.0000 \\
(0.00)\end{array}$ & $\begin{array}{l}.0000 \\
(0.00)\end{array}$ & & $\begin{array}{l}0.0000 \\
(0.00)\end{array}$ & $\begin{array}{l}0.0000 \\
(0.00)\end{array}$ & & & 2579.28 \\
\hline Switzerland & $\begin{array}{l}0.0000 \\
(0.00)\end{array}$ & $\begin{array}{c}0.0000 \\
(0.00)\end{array}$ & $\begin{array}{l}0.0000 \\
(0.00)\end{array}$ & $\begin{array}{l}0.0000 \\
(0.00)\end{array}$ & $\begin{array}{l}0.0000 \\
(0.00)\end{array}$ & $\begin{array}{l}0.0000 \\
(0.00)\end{array}$ & $\begin{array}{c}0.9916 \\
(196.67)\end{array}$ & $\begin{array}{l}0.0000 \\
(0.00)\end{array}$ & $\begin{array}{l}0.0000 \\
(0.00)\end{array}$ & $\begin{array}{l}0.0000 \\
(0.00)\end{array}$ & $\begin{array}{l}0.0000 \\
(0.00)\end{array}$ & $\begin{array}{l}0.0000 \\
(0.00)\end{array}$ & $\begin{array}{c}0.0000 \\
(0.00)\end{array}$ & $\begin{array}{l}0.0000 \\
(0.00)\end{array}$ & $\begin{array}{l}0.0000 \\
(0.00)\end{array}$ & \begin{tabular}{|c|}
0.0000 \\
$(0.00)$
\end{tabular} & $\begin{array}{l}.0000 \\
(0.00)\end{array}$ & $\begin{array}{l}0.0084 \\
(1.66)\end{array}$ & $\begin{array}{l}0.0000 \\
(0.00)\end{array}$ & $\begin{array}{l}0.0000 \\
(0.00)\end{array}$ & $\begin{array}{l}0.0000 \\
(0.00)\end{array}$ & 198.33 \\
\hline UAE & $\begin{array}{c}0.0000 \\
(0.00)\end{array}$ & $\begin{array}{l}1.0000 \\
(39.38) \\
\end{array}$ & $\begin{array}{c}0.0000 \\
(0.00)\end{array}$ & $\begin{array}{l}0.0000 \\
(0.00)\end{array}$ & $\begin{array}{l}0.0000 \\
(0.00)\end{array}$ & $\begin{array}{l}0.0000 \\
(0.00)\end{array}$ & $\begin{array}{c}0.0000 \\
(0.00) \\
\end{array}$ & $\begin{array}{l}0.0000 \\
(0.00)\end{array}$ & $\begin{array}{c}0.0000 \\
(0.00)\end{array}$ & $\begin{array}{c}0.0000 \\
(0.00) \\
\end{array}$ & $\begin{array}{c}0.0000 \\
(0.00)\end{array}$ & $\begin{array}{c}0.0000 \\
(0.00)\end{array}$ & $\begin{array}{c}0.0000 \\
(0.00)\end{array}$ & $\begin{array}{l}0.0000 \\
(0.00)\end{array}$ & $\begin{array}{l}0.0000 \\
(0.00) \\
\end{array}$ & \begin{tabular}{|c|}
0.0000 \\
$(0.00)$ \\
\end{tabular} & $\begin{array}{l}0.0000 \\
(0.00)\end{array}$ & $\begin{array}{c}0.0000 \\
(0.00)\end{array}$ & $\begin{array}{l}0.0000 \\
(0.00)\end{array}$ & $\begin{array}{c}0.0000 \\
(0.00)\end{array}$ & $\begin{array}{l}0.0000 \\
(0.00)\end{array}$ & 39.28 \\
\hline UK & $\begin{array}{l}0.0000 \\
(0.00)\end{array}$ & $\begin{array}{l}0.0000 \\
(0.00)\end{array}$ & $\begin{array}{l}0.0000 \\
(0.00)\end{array}$ & $\begin{array}{l}0.0000 \\
(0.00)\end{array}$ & $\begin{array}{l}0.0000 \\
(0.00)\end{array}$ & $\begin{array}{l}0.0000 \\
(0.00)\end{array}$ & $\begin{array}{l}0.0000 \\
(0.00)\end{array}$ & $\begin{array}{l}0.0000 \\
(0.00)\end{array}$ & $\begin{array}{c}0.1096 \\
(161.36)\end{array}$ & $\begin{array}{l}0.0000 \\
(0.00)\end{array}$ & $\begin{array}{l}0.0000 \\
(0.00)\end{array}$ & $\begin{array}{l}0.0000 \\
(0.00)\end{array}$ & $\begin{array}{l}0.0000 \\
(0.00)\end{array}$ & $\begin{array}{l}0.0000 \\
(0.00)\end{array}$ & $\begin{array}{c}0.5206 \\
(766.50)\end{array}$ & \begin{tabular}{|c|}
0.0000 \\
$(0.00)$
\end{tabular} & $\begin{array}{l}0.0000 \\
(0.00)\end{array}$ & $\begin{array}{l}0.0000 \\
(0.00)\end{array}$ & $\begin{array}{l}\mathbf{0 . 0 0 0 0} \\
(0.00)\end{array}$ & $\begin{array}{l}0.0000 \\
(0.00)\end{array}$ & $\begin{array}{c}0.3698 \\
(544.47)\end{array}$ & 1472.33 \\
\hline USA & $\begin{array}{l}0.0000 \\
(0.00)\end{array}$ & $\begin{array}{l}0.0000 \\
(0.00)\end{array}$ & $\begin{array}{c}0.0000 \\
(0.00)\end{array}$ & $\begin{array}{l}0.0000 \\
(0.00)\end{array}$ & $\begin{array}{l}0.0000 \\
(0.00)\end{array}$ & $\begin{array}{l}0.0000 \\
(0.00)\end{array}$ & $\begin{array}{l}0.0000 \\
(0.00)\end{array}$ & $\begin{array}{l}0.0000 \\
(0.00)\end{array}$ & & $\begin{array}{c}0.0000 \\
(0.00) \\
\end{array}$ & $\begin{array}{l}0.0000 \\
(0.00)\end{array}$ & $\begin{array}{l}0.0000 \\
(0.00)\end{array}$ & $\begin{array}{c}.0000 \\
(0.00) \\
\end{array}$ & $\begin{array}{c}0.0000 \\
(0.00)\end{array}$ & $\begin{array}{c}1.0000 \\
(1598.94) \\
\end{array}$ & \begin{tabular}{|c|}
0.0000 \\
$(0.00)$ \\
\end{tabular} & $\begin{array}{l}0.0000 \\
(0.00)\end{array}$ & $\begin{array}{l}0.0000 \\
(0.00)\end{array}$ & $\begin{array}{c}0.0000 \\
(0.00) \\
\end{array}$ & $\begin{array}{c}.0000 \\
(0.00)\end{array}$ & $\begin{array}{c}0.0000 \\
(0.00)\end{array}$ & 1598.94 \\
\hline Others & $\begin{array}{c}0.0000 \\
(0.00) \\
\end{array}$ & $\begin{array}{l}0.0000 \\
(0.00)\end{array}$ & $\begin{array}{c}0.0408 \\
(294.60) \\
\end{array}$ & $\begin{array}{c}0.1371 \\
(990.35) \\
\end{array}$ & $\begin{array}{l}0.0128 \\
(92.51) \\
\end{array}$ & $\begin{array}{l}0.0000 \\
(0.00)\end{array}$ & $\begin{array}{l}0.0000 \\
(0.00) \\
\end{array}$ & $\begin{array}{l}0.0050 \\
(35.93) \\
\end{array}$ & $\begin{array}{l}0.0133 \\
(96.01) \\
\end{array}$ & \begin{tabular}{|l|}
0.0074 \\
$(53.38)$ \\
\end{tabular} & $\begin{array}{l}0.0044 \\
(31.70) \\
\end{array}$ & $\begin{array}{c}0.0318 \\
(229.73) \\
\end{array}$ & \begin{tabular}{|c|}
0.2144 \\
$(1548.73)$ \\
\end{tabular} & $\begin{array}{c}0.1305 \\
(942.82) \\
\end{array}$ & $\begin{array}{c}0.0000 \\
(0.00) \\
\end{array}$ & \begin{tabular}{|c|}
0.0665 \\
$(480.70)$ \\
\end{tabular} & $\begin{array}{c}0.0000 \\
(0.00)\end{array}$ & $\begin{array}{l}0.0002 \\
(1.73)\end{array}$ & \begin{tabular}{|c|}
0.0587 \\
$(424.03)$ \\
\end{tabular} & \begin{tabular}{|c|}
0.0446 \\
$(321.98)$ \\
\end{tabular} & \begin{tabular}{|c|}
0.2325 \\
$(1679.83)$ \\
\end{tabular} & 7224.03 \\
\hline Total Export & 28677.66 & 1995.62 & 5400.34 & 6427.88 & \begin{tabular}{|l|l|}
1488.57 \\
\end{tabular} & 4768.87 & 15563.90 & 1803.36 & & \begin{tabular}{|l|}
213.97 \\
\end{tabular} & 600.93 & 296.70 & 2666.56 & 1177.54 & \begin{tabular}{|l|}
6598.32 \\
\end{tabular} & \begin{tabular}{|l|}
653.60 \\
\end{tabular} & 163.50 & 55.88 & \begin{tabular}{|l|}
439.25 \\
\end{tabular} & 432.15 & 5773.44 & \\
\hline Percentage & 105.21 & 116.38 & 145.91 & 108.16 & 81.08 & 96.80 & 112.52 & 109.19 & 136.44 & 102.87 & 116.99 & 45.98 & 92.95 & 32.86 & 189.63 & 25.34 & 82.44 & 142.26 & 29.83 & 27.03 & 79.92 & \\
\hline $\begin{array}{l}\text { \% Increase/ } \\
\text { Decrease }\end{array}$ & 5.21 & 16.38 & 45.91 & 8.16 & -18.92 & -3.20 & 12.52 & 9.19 & 36.44 & 2.87 & 16.99 & -54.02 & -7.05 & -67.14 & 89.63 & -74.66 & -17.56 & 42.26 & -70.17 & -72.97 & -20.08 & \\
\hline
\end{tabular}

Note: Figures in the parenthesis indicate average quantity of export (in tons) from India 
Table.3 Transitional probability matrix of cotton exports in the post-WTO period (1995-96 to 2012-13)

\begin{tabular}{|c|c|c|c|c|c|c|c|c|c|c|c|c|c|c|c|c|c|c|c|c|c|c|}
\hline & China & Bangladesh & Indonesia & Thailand & Viet Nam & Turkey & Japan & Malaysia & Mauritius & Nepal & Philippines & Portugal & $\begin{array}{c}\text { R. of } \\
\text { Korea } \\
\end{array}$ & Singapore & Spain & Sri Lanka & Switzerland & UAE & UK & USA & Others & $\begin{array}{c}\text { Average } \\
\text { Export }\end{array}$ \\
\hline China & \begin{tabular}{|c|}
$\mathbf{0 . 6 6 8 2}$ \\
$(190196.5)$ \\
\end{tabular} & $\begin{array}{c}0.0000 \\
(0.00) \\
\end{array}$ & \begin{tabular}{|c|}
0.0420 \\
$(11964.81)$
\end{tabular} & $\begin{array}{l}0.0000 \\
(0.00)\end{array}$ & \begin{tabular}{|c|}
0.0481 \\
$(13688.5)$ \\
\end{tabular} & \begin{tabular}{|c|}
0.0327 \\
$(9297.1)$ \\
\end{tabular} & $\begin{array}{c}0.0000 \\
(0.00)\end{array}$ & $\begin{array}{c}0.0000 \\
(0.00)\end{array}$ & $\begin{array}{c}0.0000 \\
(0.00)\end{array}$ & \begin{tabular}{|c|}
0.0000 \\
$(0.00)$ \\
\end{tabular} & $\begin{array}{c}0.0000 \\
(0.00)\end{array}$ & $\begin{array}{c}0.0000 \\
(0.00)\end{array}$ & $\begin{array}{c}0.0000 \\
(0.00) \\
\end{array}$ & $\begin{array}{c}0.0000 \\
(0.00)\end{array}$ & $\begin{array}{c}0.0000 \\
(0.00) \\
\end{array}$ & $\begin{array}{c}0.0000 \\
(0.00)\end{array}$ & $\begin{array}{c}0.0000 \\
(0.00)\end{array}$ & $\begin{array}{c}0.0008 \\
(216.28) \\
\end{array}$ & $\begin{array}{c}0.0000 \\
(0.00) \\
\end{array}$ & $\begin{array}{c}0.0000 \\
(0.00)\end{array}$ & \begin{tabular}{|c|}
0.2083 \\
$(59291.77)$ \\
\end{tabular} & 284654.83 \\
\hline Bangladesh & \begin{tabular}{|c|}
0.8778 \\
$(50857.44)$ \\
\end{tabular} & $\begin{array}{l}.0000 \\
(0.00)\end{array}$ & \begin{tabular}{|c|}
0.0000 \\
$(0.00)$ \\
\end{tabular} & $\begin{array}{c}0.1217 \\
(7053.24) \\
\end{array}$ & \begin{tabular}{|c|}
0.0000 \\
$(0.00)$ \\
\end{tabular} & $\begin{array}{c}0.0000 \\
(0.00) \\
\end{array}$ & $\begin{array}{c}0.0000 \\
(0.00)\end{array}$ & $\begin{array}{l}0.0000 \\
(0.00)\end{array}$ & $\begin{array}{c}0.0000 \\
(0.00)\end{array}$ & \begin{tabular}{|l|}
0.0000 \\
$(0.00)$ \\
\end{tabular} & $\begin{array}{c}.0000 \\
(0.00)\end{array}$ & $\begin{array}{l}.0000 \\
(0.00)\end{array}$ & \begin{tabular}{|l}
0.0004 \\
$(24.76)$ \\
\end{tabular} & $\begin{array}{c}0.0000 \\
(0.00)\end{array}$ & $\begin{array}{l}0.0000 \\
(0.00)\end{array}$ & $\begin{array}{l}0.0000 \\
(0.00)\end{array}$ & $\begin{array}{l}.0000 \\
(0.00)\end{array}$ & $\begin{array}{c}0.0000 \\
(0.00) \\
\end{array}$ & \begin{tabular}{|c|}
0.0000 \\
$(0.00)$ \\
\end{tabular} & \begin{tabular}{|c|}
0.0000 \\
$(0.00)$ \\
\end{tabular} & \begin{tabular}{|c|}
0.0000 \\
$(0.00)$ \\
\end{tabular} & 57935.44 \\
\hline Indonesia & \begin{tabular}{|c|}
0.5110 \\
$(13253.50)$
\end{tabular} & $\begin{array}{c}0.0000 \\
(0.00)\end{array}$ & $\begin{array}{c}.0000 \\
(0.00)\end{array}$ & \begin{tabular}{|c|}
0.0081 \\
$(209.07)$
\end{tabular} & $\begin{array}{c}0.0000 \\
(0.00)\end{array}$ & $\begin{array}{c}0.0000 \\
(0.00)\end{array}$ & $\begin{array}{c}0.2511 \\
(6513.21)\end{array}$ & $\begin{array}{c}0.0000 \\
(0.00)\end{array}$ & $\begin{array}{c}0.0549 \\
(1424.41)\end{array}$ & \begin{tabular}{|c|}
0.0000 \\
$(0.00)$
\end{tabular} & $\begin{array}{c}0.0226 \\
(587.33)\end{array}$ & $\begin{array}{c}.0000 \\
(0.00)\end{array}$ & \begin{tabular}{|c|}
0.0000 \\
$(0.00)$
\end{tabular} & $\begin{array}{c}0.0784 \\
(2032.56)\end{array}$ & $\begin{array}{c}0.0047 \\
(122.09)\end{array}$ & $\begin{array}{c}.0000 \\
(0.00)\end{array}$ & $\begin{array}{c}0.0000 \\
(0.00)\end{array}$ & $\begin{array}{c}.0000 \\
(0.00)\end{array}$ & \begin{tabular}{|c|}
0.0255 \\
$(660.11)$
\end{tabular} & \begin{tabular}{|c|}
0.0437 \\
$(1132.45)$
\end{tabular} & $\begin{array}{c}0.0000 \\
(0.00)\end{array}$ & 25934.72 \\
\hline Thailand & \begin{tabular}{|c|}
0.5970 \\
$(8395.54)$ \\
\end{tabular} & $\begin{array}{c}0.0000 \\
(0.00)\end{array}$ & $\begin{array}{c}0.2597 \\
(3652.59) \\
\end{array}$ & \begin{tabular}{|c|}
0.1059 \\
$(1489.61)$ \\
\end{tabular} & $\begin{array}{l}0.0000 \\
(0.00)\end{array}$ & $\begin{array}{c}.0000 \\
(0.00)\end{array}$ & $\begin{array}{c}.0000 \\
(0.00) \\
\end{array}$ & $\begin{array}{l}0.0000 \\
(0.00)\end{array}$ & $\begin{array}{c}0.0157 \\
(220.31) \\
\end{array}$ & \begin{tabular}{|c|}
0.0000 \\
$(0.00)$ \\
\end{tabular} & $\begin{array}{c}0.0000 \\
(0.00) \\
\end{array}$ & $\begin{array}{c}0.0138 \\
(193.47) \\
\end{array}$ & \begin{tabular}{|c|}
0.0000 \\
$(0.00)$ \\
\end{tabular} & $\begin{array}{l}0.0015 \\
(21.46) \\
\end{array}$ & $\begin{array}{c}0.0000 \\
(0.00)\end{array}$ & $\begin{array}{c}0.0000 \\
(0.00)\end{array}$ & $\begin{array}{l}.0000 \\
(0.00)\end{array}$ & $\begin{array}{l}0.0064 \\
(90.08)\end{array}$ & \begin{tabular}{|c|}
0.0000 \\
$(0.00)$ \\
\end{tabular} & $\begin{array}{c}0.0000 \\
(0.00) \\
\end{array}$ & $\begin{array}{l}.0000 \\
(0.00)\end{array}$ & 14063.06 \\
\hline Viet Nam & $\begin{array}{l}0.0000 \\
(0.00)\end{array}$ & $\begin{array}{c}0.8759 \\
(12931.70)\end{array}$ & $\begin{array}{l}0.0000 \\
(0.00)\end{array}$ & $\begin{array}{c}0.0000 \\
(0.00)\end{array}$ & \begin{tabular}{|c|}
0.0825 \\
$(\mathbf{1 2 1 7 . 6 2})$ \\
\end{tabular} & $\begin{array}{l}0.0000 \\
(0.00)\end{array}$ & $\begin{array}{c}0.0000 \\
(0.00)\end{array}$ & $\begin{array}{l}0.0037 \\
(54.04)\end{array}$ & $\begin{array}{c}0.0000 \\
(0.00)\end{array}$ & $\begin{array}{c}0.0000 \\
(0.00)\end{array}$ & $\begin{array}{c}0.0000 \\
(0.00)\end{array}$ & $\begin{array}{c}0.0000 \\
(0.00)\end{array}$ & $\begin{array}{c}0.0380 \\
(560.75)\end{array}$ & $\begin{array}{c}0.0000 \\
(0.00)\end{array}$ & $\begin{array}{c}0.0000 \\
(0.00)\end{array}$ & $\begin{array}{c}0.0000 \\
(0.00)\end{array}$ & $\begin{array}{c}0.0000 \\
(0.00)\end{array}$ & $\begin{array}{c}0.0000 \\
(0.00)\end{array}$ & \begin{tabular}{|c|}
0.0000 \\
$(0.00)$
\end{tabular} & $\begin{array}{c}0.0000 \\
(0.00)\end{array}$ & $\begin{array}{l}0.0000 \\
(0.00)\end{array}$ & 14764.11 \\
\hline Turkey & \begin{tabular}{|c|}
0.7719 \\
$(10423.24)$ \\
\end{tabular} & $\begin{array}{c}0.0684 \\
(924.27) \\
\end{array}$ & $\begin{array}{c}0.0211 \\
(285.05) \\
\end{array}$ & $\begin{array}{l}0.0000 \\
(0.00) \\
\end{array}$ & \begin{tabular}{|c|}
0.0383 \\
$(516.33)$ \\
\end{tabular} & $\begin{array}{c}0.0922 \\
(1244.78) \\
\end{array}$ & $\begin{array}{c}0.0000 \\
(0.00) \\
\end{array}$ & $\begin{array}{c}0.0000 \\
(0.00)\end{array}$ & $\begin{array}{l}0.0052 \\
(70.14) \\
\end{array}$ & \begin{tabular}{|l|}
0.0000 \\
$(0.00)$ \\
\end{tabular} & $\begin{array}{l}0.0000 \\
(0.00)\end{array}$ & $\begin{array}{l}.0000 \\
(0.00)\end{array}$ & $\begin{array}{l}0.0000 \\
(0.00) \\
\end{array}$ & $\begin{array}{l}0.0000 \\
(0.00)\end{array}$ & $\begin{array}{l}0.0000 \\
(0.00)\end{array}$ & $\begin{array}{l}0.0000 \\
(0.00)\end{array}$ & $\begin{array}{l}0.0000 \\
(0.00) \\
\end{array}$ & $\begin{array}{l}0.0029 \\
(39.55)\end{array}$ & \begin{tabular}{|c|}
0.0000 \\
$(0.00)$
\end{tabular} & $\begin{array}{l}0.0000 \\
(0.00)\end{array}$ & $\begin{array}{l}0.0000 \\
(0.00)\end{array}$ & 13503.56 \\
\hline Japan & \begin{tabular}{|c|}
0.0000 \\
$(0.00)$ \\
\end{tabular} & $\begin{array}{c}0.0000 \\
(0.00) \\
\end{array}$ & $\begin{array}{c}0.0000 \\
(0.00) \\
\end{array}$ & $\begin{array}{l}0.0000 \\
(0.00) \\
\end{array}$ & $\begin{array}{c}0.0000 \\
(0.00)\end{array}$ & $\begin{array}{c}0.0016 \\
(9.59) \\
\end{array}$ & \begin{tabular}{|c|}
$\mathbf{0 . 6 6 2 0}$ \\
$(\mathbf{3 8 9 3 . 0 3})$ \\
\end{tabular} & $\begin{array}{c}0.0323 \\
(189.95) \\
\end{array}$ & $\begin{array}{l}0.0000 \\
(0.00)\end{array}$ & \begin{tabular}{|c|}
0.0300 \\
$(176.47)$ \\
\end{tabular} & $\begin{array}{l}0.0000 \\
(0.00) \\
\end{array}$ & $\begin{array}{l}0.0114 \\
(66.99) \\
\end{array}$ & $\begin{array}{l}0.0000 \\
(0.00) \\
\end{array}$ & $\begin{array}{l}0.0000 \\
(0.00) \\
\end{array}$ & $\begin{array}{l}0.0000 \\
(0.00) \\
\end{array}$ & $\begin{array}{l}0.0052 \\
(30.74) \\
\end{array}$ & $\begin{array}{l}0.0003 \\
(1.64) \\
\end{array}$ & $\begin{array}{l}0.0000 \\
(0.00)\end{array}$ & \begin{tabular}{|l|}
0.0065 \\
$(38.10)$ \\
\end{tabular} & $\begin{array}{l}0.0082 \\
(48.02) \\
\end{array}$ & $\begin{array}{c}0.2425 \\
(1426.32) \\
\end{array}$ & 5880.83 \\
\hline Malaysia & $\begin{array}{l}0.0000 \\
(0.00) \\
\end{array}$ & $\begin{array}{l}0.0000 \\
(0.00) \\
\end{array}$ & $\begin{array}{l}0.0000 \\
(0.00) \\
\end{array}$ & $\begin{array}{l}0.0000 \\
(0.00) \\
\end{array}$ & $\begin{array}{l}0.0000 \\
(0.00) \\
\end{array}$ & $\begin{array}{l}0.0000 \\
(0.00) \\
\end{array}$ & \begin{tabular}{|c|}
0.7040 \\
$(3784.63)$ \\
\end{tabular} & $\begin{array}{c}0.2818 \\
(1514.80) \\
\end{array}$ & $\begin{array}{l}0.0000 \\
(0.00) \\
\end{array}$ & \begin{tabular}{|l|}
0.0134 \\
$(72.16)$ \\
\end{tabular} & $\begin{array}{l}0.0000 \\
(0.00) \\
\end{array}$ & $\begin{array}{l}0.0000 \\
(0.00)\end{array}$ & \begin{tabular}{|c|}
0.0000 \\
$(0.00)$ \\
\end{tabular} & $\begin{array}{l}0.0000 \\
(0.00)\end{array}$ & $\begin{array}{l}0.0000 \\
(0.00)\end{array}$ & $\begin{array}{l}0.0008 \\
(4.30) \\
\end{array}$ & $\begin{array}{l}0.0000 \\
(0.00) \\
\end{array}$ & $\begin{array}{l}0.0000 \\
(0.00)\end{array}$ & \begin{tabular}{|c|}
0.0000 \\
$(0.00)$ \\
\end{tabular} & $\begin{array}{l}0.0000 \\
(0.00) \\
\end{array}$ & $\begin{array}{l}0.0000 \\
(0.00) \\
\end{array}$ & $\mathbf{5 3 7 5 . 8 9}$ \\
\hline Mauritius & $\begin{array}{c}0.7975 \\
(2515.47) \\
\end{array}$ & $\begin{array}{l}0.0000 \\
(0.00)\end{array}$ & $\begin{array}{l}0.0000 \\
(0.00)\end{array}$ & $\begin{array}{l}0.0000 \\
(0.00)\end{array}$ & $\begin{array}{l}0.0000 \\
(0.00) \\
\end{array}$ & $\begin{array}{l}0.0000 \\
(0.00)\end{array}$ & $\begin{array}{c}0.0000 \\
(0.00) \\
\end{array}$ & $\begin{array}{c}0.0000 \\
(0.00) \\
\end{array}$ & $\begin{array}{r}0.1343 \\
(423.62) \\
\end{array}$ & \begin{tabular}{|c|}
0.0000 \\
$(0.00)$ \\
\end{tabular} & $\begin{array}{l}0.0000 \\
(0.00)\end{array}$ & $\begin{array}{l}0.0000 \\
(0.00)\end{array}$ & $\begin{array}{c}0.0683 \\
(215.30) \\
\end{array}$ & $\begin{array}{l}0.0000 \\
(0.00) \\
\end{array}$ & $\begin{array}{l}0.0000 \\
(0.00)\end{array}$ & $\begin{array}{l}0.0000 \\
(0.00)\end{array}$ & $\begin{array}{l}0.0000 \\
(0.00)\end{array}$ & $\begin{array}{c}.0000 \\
(0.00)\end{array}$ & \begin{tabular}{|c|}
0.0000 \\
$(0.00)$ \\
\end{tabular} & $\begin{array}{l}0.0000 \\
(0.00)\end{array}$ & $\begin{array}{l}.0000 \\
(0.00)\end{array}$ & 3154.39 \\
\hline Nepal & $\begin{array}{c}0.0000 \\
(0.00) \\
\end{array}$ & $\begin{array}{l}0.0000 \\
(0.00)\end{array}$ & $\begin{array}{l}0.0000 \\
(0.00)\end{array}$ & $\begin{array}{l}0.0000 \\
(0.00)\end{array}$ & $\begin{array}{l}0.0000 \\
(0.00)\end{array}$ & $\begin{array}{l}0.0000 \\
(0.00) \\
\end{array}$ & $\begin{array}{l}0.0000 \\
(0.00) \\
\end{array}$ & $\begin{array}{c}0.9095 \\
(875.35) \\
\end{array}$ & $\begin{array}{l}0.0000 \\
(0.00)\end{array}$ & \begin{tabular}{|c|}
$\begin{array}{c}0.0000 \\
(0.00)\end{array}$ \\
\end{tabular} & $\begin{array}{c}0.0000 \\
(0.00) \\
\end{array}$ & $\begin{array}{l}0.0000 \\
(0.00)\end{array}$ & \begin{tabular}{|l|}
0.0905 \\
$(87.09)$ \\
\end{tabular} & $\begin{array}{l}0.0000 \\
(0.00)\end{array}$ & $\begin{array}{l}0.0000 \\
(0.00)\end{array}$ & $\begin{array}{c}0.0000 \\
(0.00)\end{array}$ & $\begin{array}{l}0.0000 \\
(0.00) \\
\end{array}$ & $\begin{array}{c}0.0000 \\
(0.00)\end{array}$ & \begin{tabular}{|c|}
0.0000 \\
$(0.00)$ \\
\end{tabular} & $\begin{array}{l}0.0000 \\
(0.00) \\
\end{array}$ & $\begin{array}{l}0.0000 \\
(0.00) \\
\end{array}$ & 962.44 \\
\hline Philippines & $\begin{array}{c}0.0000 \\
(0.00)\end{array}$ & $\begin{array}{c}0.0000 \\
(0.00)\end{array}$ & $\begin{array}{c}0.0000 \\
(0.00)\end{array}$ & $\begin{array}{c}0.4930 \\
(541.55)\end{array}$ & $\begin{array}{l}0.0000 \\
(0.00)\end{array}$ & $\begin{array}{c}0.0000 \\
(0.00)\end{array}$ & $\begin{array}{c}0.0000 \\
(0.00)\end{array}$ & $\begin{array}{l}0.0000 \\
(0.00)\end{array}$ & $\begin{array}{c}0.0000 \\
(0.00)\end{array}$ & $\begin{array}{c}0.0000 \\
(0.00)\end{array}$ & $\begin{array}{c}0.2284 \\
(250.86)\end{array}$ & $\begin{array}{l}0.0846 \\
(92.97)\end{array}$ & $\begin{array}{c}0.0000 \\
(0.00)\end{array}$ & $\begin{array}{l}0.0000 \\
(0.00)\end{array}$ & $\begin{array}{c}0.0000 \\
(0.00)\end{array}$ & $\begin{array}{c}0.1354 \\
(148.77)\end{array}$ & $\begin{array}{l}0.0315 \\
(34.62)\end{array}$ & $\begin{array}{c}0.0000 \\
(0.00)\end{array}$ & $\begin{array}{c}0.0000 \\
(0.00)\end{array}$ & $\begin{array}{l}0.0270 \\
(29.68)\end{array}$ & $\begin{array}{c}0.0000 \\
(0.00)\end{array}$ & 1098.44 \\
\hline Portugal & $\begin{array}{c}0.9263 \\
(436.47)\end{array}$ & $\begin{array}{c}0.0000 \\
(0.00)\end{array}$ & $\begin{array}{c}0.0000 \\
(0.00)\end{array}$ & $\begin{array}{c}0.0000 \\
(0.00)\end{array}$ & $\begin{array}{c}0.0000 \\
(0.00)\end{array}$ & $\begin{array}{c}0.0000 \\
(0.00)\end{array}$ & $\begin{array}{c}0.0000 \\
(0.00)\end{array}$ & $\begin{array}{c}0.0000 \\
(0.00)\end{array}$ & $\begin{array}{c}0.0000 \\
(0.00)\end{array}$ & $\begin{array}{c}0.0000 \\
(0.00)\end{array}$ & $\begin{array}{l}0.0710 \\
(33.48)\end{array}$ & $\begin{array}{c}.0000 \\
(0.00)\end{array}$ & $\begin{array}{c}0.0000 \\
(0.00)\end{array}$ & $\begin{array}{c}0.0000 \\
(0.00)\end{array}$ & $\begin{array}{l}0.0000 \\
(0.00)\end{array}$ & $\begin{array}{c}0.0000 \\
(0.00)\end{array}$ & $\begin{array}{c}0.0000 \\
(0.00)\end{array}$ & $\begin{array}{l}0.0027 \\
(1.28)\end{array}$ & \begin{tabular}{|c|}
0.0000 \\
$(0.00)$
\end{tabular} & $\begin{array}{l}0.0000 \\
(0.00)\end{array}$ & $\begin{array}{l}0.0000 \\
(0.00)\end{array}$ & 471.22 \\
\hline \begin{tabular}{|l|} 
Republic of \\
Korea
\end{tabular} & $\begin{array}{c}0.0000 \\
(0.00) \\
\end{array}$ & $\begin{array}{l}0.0000 \\
(0.00)\end{array}$ & $\begin{array}{c}0.9575 \\
(2024.21) \\
\end{array}$ & $\begin{array}{l}0.0000 \\
(0.00)\end{array}$ & $\begin{array}{l}0.0000 \\
(0.00)\end{array}$ & $\begin{array}{l}0.0000 \\
(0.00)\end{array}$ & $\begin{array}{l}0.0000 \\
(0.00) \\
\end{array}$ & $\begin{array}{l}0.0000 \\
(0.00)\end{array}$ & $\begin{array}{l}0.0000 \\
(0.00) \\
\end{array}$ & \begin{tabular}{|l|}
0.0000 \\
$(0.00)$
\end{tabular} & $\begin{array}{l}0.0425 \\
(89.79) \\
\end{array}$ & $\begin{array}{l}0.0000 \\
(0.00)\end{array}$ & \begin{tabular}{|c|}
0.0000 \\
$(0.00)$ \\
\end{tabular} & $\begin{array}{l}0.0000 \\
(0.00) \\
\end{array}$ & $\begin{array}{l}0.0000 \\
(0.00) \\
\end{array}$ & $\begin{array}{l}0.0000 \\
(0.00)\end{array}$ & $\begin{array}{l}0.0000 \\
(0.00)\end{array}$ & $\begin{array}{c}0.0000 \\
(0.00)\end{array}$ & \begin{tabular}{|c|}
0.0000 \\
$(0.00)$ \\
\end{tabular} & $\begin{array}{l}0.0000 \\
(0.00)\end{array}$ & $\begin{array}{l}0.0000 \\
(0.00)\end{array}$ & 2114.00 \\
\hline Singapore & \begin{tabular}{|c|}
0.9330 \\
$(1968.34)$
\end{tabular} & $\begin{array}{l}0.0000 \\
(0.00)\end{array}$ & $\begin{array}{l}0.0000 \\
(0.00)\end{array}$ & $\begin{array}{l}0.0000 \\
(0.00)\end{array}$ & $\begin{array}{l}0.0000 \\
(0.00)\end{array}$ & $\begin{array}{l}0.0000 \\
(0.00)\end{array}$ & $\begin{array}{l}0.0000 \\
(0.00)\end{array}$ & $\begin{array}{l}0.0000 \\
(0.00)\end{array}$ & $\begin{array}{l}0.0000 \\
(0.00)\end{array}$ & \begin{tabular}{|l|}
0.0000 \\
$(0.00)$ \\
\end{tabular} & $\begin{array}{l}0.0000 \\
(0.00)\end{array}$ & $\begin{array}{l}0.0000 \\
(0.00)\end{array}$ & \begin{tabular}{|l|}
0.0000 \\
$(0.00)$ \\
\end{tabular} & $\begin{array}{c}.0000 \\
(0.00)\end{array}$ & $\begin{array}{l}0.0000 \\
(0.00)\end{array}$ & $\begin{array}{l}0.0000 \\
(0.00)\end{array}$ & $\begin{array}{l}0.0000 \\
(0.00) \\
\end{array}$ & $\begin{array}{c}0.0670 \\
(141.33) \\
\end{array}$ & \begin{tabular}{|l|}
0.0000 \\
$(0.00)$ \\
\end{tabular} & $\begin{array}{l}0.0000 \\
(0.00) \\
\end{array}$ & $\begin{array}{l}0.0000 \\
(0.00) \\
\end{array}$ & 2109.67 \\
\hline Spain & $\begin{array}{l}0.0000 \\
(0.00) \\
\end{array}$ & $\begin{array}{c}0.0000 \\
(0.00)\end{array}$ & $\begin{array}{c}0.0000 \\
(0.00)\end{array}$ & $\begin{array}{c}0.0000 \\
(0.00)\end{array}$ & $\begin{array}{c}0.0000 \\
(0.00)\end{array}$ & $\begin{array}{l}0.3915 \\
(15.38) \\
\end{array}$ & $\begin{array}{c}0.0000 \\
(0.00) \\
\end{array}$ & $\begin{array}{c}0.0000 \\
(0.00)\end{array}$ & $\begin{array}{c}0.0000 \\
(0.00)\end{array}$ & \begin{tabular}{|c|}
0.0000 \\
$(0.00)$ \\
\end{tabular} & $\begin{array}{c}0.0000 \\
(0.00) \\
\end{array}$ & $\begin{array}{c}0.0000 \\
(0.00)\end{array}$ & $\begin{array}{c}0.0000 \\
(0.00) \\
\end{array}$ & $\begin{array}{c}0.0000 \\
(0.00)\end{array}$ & $\begin{array}{c}0.0000 \\
(0.00)\end{array}$ & $\begin{array}{c}0.0000 \\
(0.00)\end{array}$ & $\begin{array}{l}0.6085 \\
(23.90) \\
\end{array}$ & $\begin{array}{c}0.0000 \\
(0.00) \\
\end{array}$ & $\begin{array}{c}0.0000 \\
(0.00) \\
\end{array}$ & $\begin{array}{c}0.0000 \\
(0.00) \\
\end{array}$ & $\begin{array}{c}0.0000 \\
(0.00)\end{array}$ & 39.28 \\
\hline Sri Lanka & $\begin{array}{c}0.0000 \\
(0.00)\end{array}$ & $\begin{array}{c}0.0000 \\
(0.00)\end{array}$ & $\begin{array}{c}0.0000 \\
(0.00)\end{array}$ & $\begin{array}{c}0.0000 \\
(0.00)\end{array}$ & $\begin{array}{c}0.0000 \\
(0.00)\end{array}$ & $\begin{array}{c}0.0000 \\
(0.00)\end{array}$ & $\begin{array}{c}1.0000 \\
(267.61)\end{array}$ & $\begin{array}{c}0.0000 \\
(0.00)\end{array}$ & $\begin{array}{c}0.0000 \\
(0.00)\end{array}$ & \begin{tabular}{|c|}
0.0000 \\
$(0.00)$
\end{tabular} & $\begin{array}{c}0.0000 \\
(0.00)\end{array}$ & $\begin{array}{c}0.0000 \\
(0.00)\end{array}$ & \begin{tabular}{|c|}
0.0000 \\
$(0.00)$
\end{tabular} & $\begin{array}{c}0.0000 \\
(0.00)\end{array}$ & $\begin{array}{c}0.0000 \\
(0.00)\end{array}$ & $\begin{array}{c}0.0000 \\
(0.00)\end{array}$ & $\begin{array}{c}0.0000 \\
(0.00)\end{array}$ & $\begin{array}{c}0.0000 \\
(0.00)\end{array}$ & $\begin{array}{c}0.0000 \\
(0.00)\end{array}$ & $\begin{array}{c}0.0000 \\
(0.00)\end{array}$ & $\begin{array}{c}0.0000 \\
(0.00)\end{array}$ & 267.61 \\
\hline Switzerland & $\begin{array}{c}0.0000 \\
(0.00)\end{array}$ & $\begin{array}{c}0.0000 \\
(0.00)\end{array}$ & $\begin{array}{c}0.0000 \\
(0.00)\end{array}$ & $\begin{array}{c}0.0000 \\
(0.00)\end{array}$ & $\begin{array}{c}0.0000 \\
(0.00)\end{array}$ & $\begin{array}{c}0.0000 \\
(0.00)\end{array}$ & $\begin{array}{l}0.1097 \\
(14.92)\end{array}$ & $\begin{array}{c}0.0000 \\
(0.00)\end{array}$ & $\begin{array}{c}0.0000 \\
(0.00)\end{array}$ & $\begin{array}{c}0.0000 \\
(0.00)\end{array}$ & $\begin{array}{c}0.0000 \\
(0.00)\end{array}$ & $\begin{array}{c}0.0000 \\
(0.00)\end{array}$ & $\begin{array}{c}0.0000 \\
(0.00)\end{array}$ & $\begin{array}{c}0.0000 \\
(0.00)\end{array}$ & $\begin{array}{l}0.0000 \\
(0.00)\end{array}$ & $\begin{array}{c}0.8903 \\
(121.03)\end{array}$ & $\begin{array}{c}\mathbf{0 . 0 0 0 0} \\
(\mathbf{0 . 0 0 )}\end{array}$ & $\begin{array}{c}0.0000 \\
(0.00)\end{array}$ & $\begin{array}{c}0.0000 \\
(0.00)\end{array}$ & $\begin{array}{c}0.0000 \\
(0.00)\end{array}$ & $\begin{array}{c}0.0000 \\
(0.00)\end{array}$ & 135.94 \\
\hline UAE & $\begin{array}{c}0.0000 \\
(0.00)\end{array}$ & $\begin{array}{c}1.0000 \\
(1135.17)\end{array}$ & $\begin{array}{c}0.0000 \\
(0.00)\end{array}$ & $\begin{array}{c}0.0000 \\
(0.00)\end{array}$ & $\begin{array}{c}0.0000 \\
(0.00)\end{array}$ & $\begin{array}{c}0.0000 \\
(0.00)\end{array}$ & $\begin{array}{c}0.0000 \\
(0.00)\end{array}$ & $\begin{array}{c}0.0000 \\
(0.00)\end{array}$ & $\begin{array}{c}0.0000 \\
(0.00)\end{array}$ & $\begin{array}{c}0.0000 \\
(0.00)\end{array}$ & $\begin{array}{c}0.0000 \\
(0.00)\end{array}$ & $\begin{array}{c}0.0000 \\
(0.00)\end{array}$ & $\begin{array}{c}0.0000 \\
(0.00)\end{array}$ & $\begin{array}{c}0.0000 \\
(0.00)\end{array}$ & $\begin{array}{c}0.0000 \\
(0.00)\end{array}$ & $\begin{array}{l}0.0000 \\
(0.00)\end{array}$ & $\begin{array}{c}0.0000 \\
(0.00)\end{array}$ & $\begin{array}{c}0.0000 \\
(0.00)\end{array}$ & $\begin{array}{c}0.0000 \\
(0.00)\end{array}$ & $\begin{array}{c}0.0000 \\
(0.00)\end{array}$ & $\begin{array}{c}0.0000 \\
(0.00)\end{array}$ & 1135.17 \\
\hline UK & $\begin{array}{l}0.0000 \\
(0.00)\end{array}$ & $\begin{array}{c}0.0000 \\
(0.00)\end{array}$ & $\begin{array}{c}0.0000 \\
(0.00)\end{array}$ & $\begin{array}{c}0.4025 \\
(257.26) \\
\end{array}$ & $\begin{array}{l}0.0000 \\
(0.00)\end{array}$ & $\begin{array}{l}0.0000 \\
(0.00)\end{array}$ & $\begin{array}{l}0.0000 \\
(0.00)\end{array}$ & $\begin{array}{l}0.0000 \\
(0.00)\end{array}$ & $\begin{array}{l}.0000 \\
(0.00)\end{array}$ & \begin{tabular}{|l|}
0.0000 \\
$(0.00)$
\end{tabular} & $\begin{array}{l}0.0000 \\
(0.00)\end{array}$ & $\begin{array}{l}0.0000 \\
(0.00)\end{array}$ & \begin{tabular}{|c|}
0.0000 \\
$(0.00)$ \\
\end{tabular} & $\begin{array}{l}0.0226 \\
(14.46)\end{array}$ & $\begin{array}{l}0.0075 \\
(4.80)\end{array}$ & $\begin{array}{l}0.0000 \\
(0.00)\end{array}$ & $\begin{array}{l}0.0000 \\
(0.00)\end{array}$ & $\begin{array}{c}.0000 \\
(0.00)\end{array}$ & \begin{tabular}{|c|}
0.0126 \\
$(8.06)$ \\
\end{tabular} & $\begin{array}{l}0.0959 \\
(61.31) \\
\end{array}$ & $\begin{array}{c}0.4589 \\
(293.33) \\
\end{array}$ & 639.22 \\
\hline USA & $\begin{array}{l}0.0000 \\
(0.00)\end{array}$ & $\begin{array}{c}0.0000 \\
(0.00)\end{array}$ & $\begin{array}{c}0.3810 \\
(377.26)\end{array}$ & $\begin{array}{l}0.0000 \\
(0.00)\end{array}$ & $\begin{array}{l}0.0000 \\
(0.00)\end{array}$ & $\begin{array}{l}0.0296 \\
(29.32)\end{array}$ & $\begin{array}{l}0.0000 \\
(0.00)\end{array}$ & $\begin{array}{c}0.0000 \\
(0.00)\end{array}$ & $\begin{array}{l}0.0000 \\
(0.00)\end{array}$ & \begin{tabular}{|l|}
0.0000 \\
$(0.00)$
\end{tabular} & $\begin{array}{l}0.0000 \\
(0.00)\end{array}$ & $\begin{array}{l}0.0000 \\
(0.00)\end{array}$ & $\begin{array}{c}0.2859 \\
(283.12) \\
\end{array}$ & $\begin{array}{l}0.0000 \\
(0.00)\end{array}$ & $\begin{array}{l}0.0000 \\
(0.00)\end{array}$ & $\begin{array}{l}0.0382 \\
(37.81)\end{array}$ & $\begin{array}{l}0.0088 \\
(8.69)\end{array}$ & $\begin{array}{c}0.0000 \\
(0.00)\end{array}$ & $\begin{array}{c}0.2080 \\
(206.01) \\
\end{array}$ & $\begin{array}{l}.0485 \\
(48.01) \\
\end{array}$ & $\begin{array}{l}0.0000 \\
(0.00) \\
\end{array}$ & 990.22 \\
\hline Others & $\begin{array}{l}0.0000 \\
(0.00) \\
\end{array}$ & $\begin{array}{c}0.4152 \\
(32098.12) \\
\end{array}$ & $\begin{array}{c}0.0429 \\
(3313.46) \\
\end{array}$ & \begin{tabular}{|c|}
0.1009 \\
$(7804.77)$ \\
\end{tabular} & \begin{tabular}{|c|}
0.0109 \\
$(840.79)$ \\
\end{tabular} & \begin{tabular}{|c|}
0.0100 \\
$(773.70)$ \\
\end{tabular} & $\begin{array}{l}0.0000 \\
(0.00) \\
\end{array}$ & $\begin{array}{c}0.0386 \\
(2987.59) \\
\end{array}$ & $\begin{array}{l}0.0000 \\
(0.00)\end{array}$ & \begin{tabular}{|c|}
0.0017 \\
$(128.55)$ \\
\end{tabular} & $\begin{array}{l}0.0000 \\
(0.00)\end{array}$ & $\begin{array}{l}0.0000 \\
(0.00)\end{array}$ & \begin{tabular}{|c|}
0.0000 \\
$(0.00)$ \\
\end{tabular} & $\begin{array}{l}0.0002 \\
(16.23) \\
\end{array}$ & $\begin{array}{l}0.0000 \\
(0.00) \\
\end{array}$ & $\begin{array}{l}0.0000 \\
(0.00)\end{array}$ & $\begin{array}{l}0.0000 \\
(0.00)\end{array}$ & $\begin{array}{l}0.0000 \\
(0.00)\end{array}$ & \begin{tabular}{|c|}
0.0000 \\
$(0.00)$ \\
\end{tabular} & $\begin{array}{l}0.0000 \\
(0.00)\end{array}$ & \begin{tabular}{|c|}
$\mathbf{0 . 3 7 9 6}$ \\
$(29350.67)$ \\
\end{tabular} & 77313.89 \\
\hline \begin{tabular}{|l|} 
Total \\
Export
\end{tabular} & 278046.46 & 47089.26 & 21617.38 & 17355.50 & 16263.39 & 11369.82 & 14473.40 & 5621.73 & 2138.48 & 377.17 & 961.45 & 353.43 & 1171.02 & 2084.71 & 126.89 & 342.65 & 68.85 & 488.51 & 912.28 & 1319.47 & 90362.09 & \\
\hline Percentage & 97.68 & 81.28 & 83.35 & 123.41 & 110.15 & 84.20 & 246.11 & 104.57 & 67.79 & 39.19 & 87.53 & 75.00 & 55.39 & 98.82 & 323.06 & 128.04 & 50.64 & 43.03 & 142.72 & 133.25 & 116.88 & \\
\hline$\left|\begin{array}{ll}\% & \text { Increase } \\
\text { Decrease }\end{array}\right|$ & -2.32 & -18.72 & -16.65 & 23.41 & 10.15 & -15.80 & 146.11 & 4.57 & -32.21 & -60.81 & -12.47 & -25.00 & -44.61 & -1.18 & 223.06 & 28.04 & -49.36 & -56.97 & 42.72 & 33.25 & 16.88 & \\
\hline
\end{tabular}

Note: Figures in the parenthesis indicate average quantity of export (in tons) from India 
Table.5 Transitional probability matrix of cotton imports in the pre-WTO period (1977-78 to 1994-95)

\begin{tabular}{|c|c|c|c|c|c|c|c|c|c|c|c|c|c|c|c|c|c|c|c|c|c|c|}
\hline & Australia & Benin & $\begin{array}{c}\text { Burkina } \\
\text { Faso }\end{array}$ & $\begin{array}{c}\text { Cote } \\
\text { deIvoire }\end{array}$ & Egypt & Mali & Pakistan & Sudan & Tanzania & USA & Uzbekistan & Cameroon & China & Israel & Malaysia & $\begin{array}{c}\text { Russian } \\
\text { Federation } \\
\end{array}$ & Switzerland & Turkey & $\begin{array}{c}\text { Turk } \\
\text { menistan }\end{array}$ & Austria & Other & $\begin{array}{l}\text { Average } \\
\text { Import }\end{array}$ \\
\hline Australia & $\begin{array}{l}\mathbf{0 . 0 0 0 0} \\
(0.00)\end{array}$ & $\begin{array}{c}0.0000 \\
(0.00)\end{array}$ & $\begin{array}{c}0.0000 \\
(0.00)\end{array}$ & $\begin{array}{c}0.0000 \\
(0.00)\end{array}$ & $\begin{array}{l}0.0000 \\
(0.00)\end{array}$ & $\begin{array}{l}0.0000 \\
(0.00)\end{array}$ & $\begin{array}{l}0.0000 \\
(0.00)\end{array}$ & $\begin{array}{l}0.0155 \\
(35.9)\end{array}$ & $\begin{array}{c}0.0516 \\
(117.13)\end{array}$ & \begin{tabular}{|l|}
0.2698 \\
$(612.4)$
\end{tabular} & $\begin{array}{l}0.0000 \\
(0.00)\end{array}$ & $\begin{array}{l}0.0000 \\
(0.00)\end{array}$ & $\begin{array}{l}0.0364 \\
(82.63)\end{array}$ & $\begin{array}{l}0.0046 \\
(10.33)\end{array}$ & $\begin{array}{l}0.0005 \\
(1.13)\end{array}$ & $\begin{array}{c}0.0028 \\
(6.42)\end{array}$ & $\begin{array}{l}0.0000 \\
(0.00)\end{array}$ & $\begin{array}{l}0.0052 \\
(11.75)\end{array}$ & $\begin{array}{c}0.0000 \\
(0.00)\end{array}$ & $\begin{array}{l}0.0000 \\
(0.00)\end{array}$ & \begin{tabular}{|c|}
0.6136 \\
$(1392.64)$
\end{tabular} & 2269.78 \\
\hline Benin & $\begin{array}{c}0.6610 \\
(764.99) \\
\end{array}$ & $\begin{array}{l}.0000 \\
(0.00)\end{array}$ & $\begin{array}{l}0.0000 \\
(0.00)\end{array}$ & $\begin{array}{l}0.0000 \\
(0.00)\end{array}$ & $\begin{array}{c}0.0000 \\
(0.00) \\
\end{array}$ & $\begin{array}{l}0.0000 \\
(0.00) \\
\end{array}$ & $\begin{array}{l}0.0000 \\
(0.00)\end{array}$ & $\begin{array}{l}.0000 \\
(0.00)\end{array}$ & $\begin{array}{l}0.0000 \\
(0.00)\end{array}$ & $\begin{array}{l}0.3390 \\
(392.4) \\
\end{array}$ & $\begin{array}{l}0.0000 \\
(0.00)\end{array}$ & $\begin{array}{l}0.0000 \\
(0.00)\end{array}$ & $\begin{array}{l}0.0000 \\
(0.00)\end{array}$ & $\begin{array}{l}0.0000 \\
(0.00) \\
\end{array}$ & $\begin{array}{c}.0000 \\
(0.00)\end{array}$ & $\begin{array}{l}0.0000 \\
(0.00)\end{array}$ & $\begin{array}{l}0.0000 \\
(0.00)\end{array}$ & $\begin{array}{l}0.0000 \\
(0.00) \\
\end{array}$ & $\begin{array}{c}0.0000 \\
(0.00)\end{array}$ & $\begin{array}{l}0.0000 \\
(0.00)\end{array}$ & \begin{tabular}{|c|}
0.0000 \\
$(0.00)$ \\
\end{tabular} & 1157.39 \\
\hline Burkina Faso & $\begin{array}{c}0.0000 \\
(0.00)\end{array}$ & $\begin{array}{c}1.0000 \\
(544.39) \\
\end{array}$ & $\begin{array}{c}0.0000 \\
(0.00)\end{array}$ & $\begin{array}{c}0.0000 \\
(0.00)\end{array}$ & $\begin{array}{c}0.0000 \\
(0.00)\end{array}$ & $\begin{array}{c}0.0000 \\
(0.00)\end{array}$ & $\begin{array}{l}0.0000 \\
(0.00)\end{array}$ & $\begin{array}{c}0.0000 \\
(0.00)\end{array}$ & $\begin{array}{c}0.0000 \\
(0.00)\end{array}$ & $\begin{array}{c}0.0000 \\
(0.00)\end{array}$ & $\begin{array}{c}0.0000 \\
(0.00)\end{array}$ & $\begin{array}{c}0.0000 \\
(0.00)\end{array}$ & $\begin{array}{c}0.0000 \\
(0.00)\end{array}$ & $\begin{array}{c}0.0000 \\
(0.00)\end{array}$ & $\begin{array}{c}0.0000 \\
(0.00)\end{array}$ & $\begin{array}{c}0.0000 \\
(0.00)\end{array}$ & $\begin{array}{c}0.0000 \\
(0.00)\end{array}$ & $\begin{array}{c}0.0000 \\
(0.00)\end{array}$ & $\begin{array}{c}0.0000 \\
(0.00)\end{array}$ & $\begin{array}{l}0.0000 \\
(0.00)\end{array}$ & $\begin{array}{c}0.0000 \\
(0.00)\end{array}$ & 544.39 \\
\hline Cote deIvoire & $\begin{array}{l}0.0000 \\
(0.00)\end{array}$ & $\begin{array}{l}0.0000 \\
(0.00)\end{array}$ & $\begin{array}{l}0.0000 \\
(0.00)\end{array}$ & $\begin{array}{c}\mathbf{0 . 0 0 0 0} \\
(0.00)\end{array}$ & $\begin{array}{c}0.0000 \\
(0.00)\end{array}$ & $\begin{array}{c}0.0000 \\
(0.00)\end{array}$ & $\begin{array}{l}0.0000 \\
(0.00)\end{array}$ & $\begin{array}{l}0.0000 \\
(0.00)\end{array}$ & $\begin{array}{c}0.0000 \\
(0.00)\end{array}$ & $\begin{array}{c}0.0000 \\
(0.00)\end{array}$ & $\begin{array}{c}0.0000 \\
(0.00)\end{array}$ & $\begin{array}{c}0.1280 \\
(103.80) \\
\end{array}$ & $\begin{array}{c}0.1728 \\
(140.11) \\
\end{array}$ & \begin{tabular}{|l|}
0.1149 \\
$(93.18)$ \\
\end{tabular} & $\begin{array}{l}0.0224 \\
(18.12)\end{array}$ & $\begin{array}{c}0.1510 \\
(122.39) \\
\end{array}$ & $\begin{array}{c}0.4108 \\
(333.06)\end{array}$ & $\begin{array}{c}0.0000 \\
(0.00)\end{array}$ & $\begin{array}{c}0.0000 \\
(0.00)\end{array}$ & $\begin{array}{c}0.0000 \\
(0.00)\end{array}$ & $\begin{array}{c}0.0000 \\
(0.00)\end{array}$ & 810.67 \\
\hline Egypt & $\begin{array}{l}0.0000 \\
(0.00)\end{array}$ & $\begin{array}{c}0.0000 \\
(0.00)\end{array}$ & $\begin{array}{l}0.0000 \\
(0.00)\end{array}$ & $\begin{array}{c}0.0000 \\
(0.00)\end{array}$ & $\begin{array}{c}0.0000 \\
(0.00)\end{array}$ & $\begin{array}{l}0.0013 \\
(2.35) \\
\end{array}$ & $\begin{array}{c}0.0000 \\
(0.00)\end{array}$ & \begin{tabular}{|c|}
0.8612 \\
$(1567.19)$ \\
\end{tabular} & $\begin{array}{c}0.0000 \\
(0.00)\end{array}$ & $\begin{array}{l}0.1375 \\
(250.2) \\
\end{array}$ & $\begin{array}{c}0.0000 \\
(0.00)\end{array}$ & $\begin{array}{c}0.0000 \\
(0.00)\end{array}$ & $\begin{array}{c}0.0000 \\
(0.00)\end{array}$ & $\begin{array}{c}0.0000 \\
(0.00)\end{array}$ & $\begin{array}{c}0.0000 \\
(0.00)\end{array}$ & $\begin{array}{c}0.0000 \\
(0.00)\end{array}$ & $\begin{array}{c}0.0000 \\
(0.00)\end{array}$ & $\begin{array}{c}0.0000 \\
(0.00)\end{array}$ & $\begin{array}{c}0.0000 \\
(0.00)\end{array}$ & $\begin{array}{c}0.0000 \\
(0.00)\end{array}$ & $\begin{array}{l}0.0000 \\
(0.00)\end{array}$ & 1819.78 \\
\hline Mali & $\begin{array}{c}0.0000 \\
(0.00)\end{array}$ & $\begin{array}{c}0.0000 \\
(0.00)\end{array}$ & $\begin{array}{l}0.0000 \\
(0.00)\end{array}$ & $\begin{array}{c}0.0000 \\
(0.00)\end{array}$ & $\begin{array}{c}0.0000 \\
(0.00)\end{array}$ & $\begin{array}{l}0.0000 \\
(0.00)\end{array}$ & $\begin{array}{c}0.0000 \\
(0.00)\end{array}$ & \begin{tabular}{|c|}
1.0000 \\
$(559.44)$
\end{tabular} & $\begin{array}{l}0.0000 \\
(0.00)\end{array}$ & $\begin{array}{c}0.0000 \\
(0.00)\end{array}$ & $\begin{array}{c}0.0000 \\
(0.00)\end{array}$ & $\begin{array}{c}0.0000 \\
(0.00)\end{array}$ & $\begin{array}{c}0.0000 \\
(0.00)\end{array}$ & $\begin{array}{c}0.0000 \\
(0.00)\end{array}$ & $\begin{array}{c}0.0000 \\
(0.00)\end{array}$ & $\begin{array}{c}0.0000 \\
(0.00)\end{array}$ & $\begin{array}{l}0.0000 \\
(0.00)\end{array}$ & $\begin{array}{c}0.0000 \\
(0.00)\end{array}$ & $\begin{array}{c}0.0000 \\
(0.00)\end{array}$ & $\begin{array}{c}0.0000 \\
(0.00)\end{array}$ & $\begin{array}{c}0.0000 \\
(0.00)\end{array}$ & 559.44 \\
\hline Pakistan & $\begin{array}{c}0.1917 \\
(1036.53)\end{array}$ & $\begin{array}{c}0.0000 \\
(0.00)\end{array}$ & $\begin{array}{l}0.0000 \\
(0.00)\end{array}$ & $\begin{array}{c}0.2507 \\
(1355.55)\end{array}$ & $\begin{array}{l}0.0000 \\
(0.00)\end{array}$ & $\begin{array}{l}0.0000 \\
(0.00)\end{array}$ & $\begin{array}{c}0.1862 \\
(\mathbf{1 0 0 6 . 7 0})\end{array}$ & \begin{tabular}{|c|}
0.0406 \\
$(219.53)$
\end{tabular} & $\begin{array}{l}0.0000 \\
(0.00)\end{array}$ & \begin{tabular}{|l|}
0.1370 \\
$(740.8)$
\end{tabular} & $\begin{array}{c}0.0000 \\
(0.00)\end{array}$ & $\begin{array}{c}0.0000 \\
(0.00)\end{array}$ & $\begin{array}{l}0.0076 \\
(40.95)\end{array}$ & $\begin{array}{c}0.0000 \\
(0.00)\end{array}$ & $\begin{array}{c}0.0000 \\
(0.00)\end{array}$ & $\begin{array}{l}0.0029 \\
(15.64)\end{array}$ & $\begin{array}{l}0.0000 \\
(0.00)\end{array}$ & $\begin{array}{c}0.0470 \\
(254.13)\end{array}$ & $\begin{array}{c}0.0348 \\
(188.37)\end{array}$ & \begin{tabular}{|c|}
0.0000 \\
$(0.00)$
\end{tabular} & $\begin{array}{c}0.1015 \\
(548.88)\end{array}$ & 5407.06 \\
\hline Sudan & $\begin{array}{l}0.0419 \\
(92.64)\end{array}$ & $\begin{array}{c}0.0000 \\
(0.00)\end{array}$ & $\begin{array}{l}0.0000 \\
(0.00)\end{array}$ & $\begin{array}{c}0.1118 \\
(247.18)\end{array}$ & $\begin{array}{l}0.0000 \\
(0.00)\end{array}$ & $\begin{array}{l}0.0004 \\
(0.88)\end{array}$ & $\begin{array}{c}0.4712 \\
(1041.80)\end{array}$ & \begin{tabular}{|c|}
$\mathbf{0 . 2 7 7 1}$ \\
$(612.65)$
\end{tabular} & $\begin{array}{l}0.0000 \\
(0.00)\end{array}$ & \begin{tabular}{|l|}
0.0295 \\
$(65.26)$
\end{tabular} & $\begin{array}{c}0.0000 \\
(0.00)\end{array}$ & $\begin{array}{c}0.0000 \\
(0.00)\end{array}$ & $\begin{array}{c}0.0000 \\
(0.00)\end{array}$ & $\begin{array}{c}0.0000 \\
(0.00)\end{array}$ & $\begin{array}{c}0.0000 \\
(0.00)\end{array}$ & $\begin{array}{c}0.0000 \\
(0.00)\end{array}$ & $\begin{array}{l}0.0151 \\
(33.39)\end{array}$ & $\begin{array}{c}0.0000 \\
(0.00)\end{array}$ & $\begin{array}{c}0.0000 \\
(0.00)\end{array}$ & \begin{tabular}{|c|}
0.0000 \\
$(0.00)$
\end{tabular} & $\begin{array}{c}0.0530 \\
(117.15)\end{array}$ & 2210.94 \\
\hline Tanzania & $\begin{array}{l}0.0000 \\
(0.00)\end{array}$ & $\begin{array}{l}0.1328 \\
(37.68) \\
\end{array}$ & $\begin{array}{c}0.7473 \\
(212.12) \\
\end{array}$ & $\begin{array}{l}0.0000 \\
(0.00)\end{array}$ & $\begin{array}{c}0.0000 \\
(0.00) \\
\end{array}$ & $\begin{array}{l}0.1199 \\
(34.03) \\
\end{array}$ & $\begin{array}{c}0.0000 \\
(0.00)\end{array}$ & \begin{tabular}{|c|}
0.0000 \\
$(0.00)$ \\
\end{tabular} & $\begin{array}{l}.0000 \\
(0.00)\end{array}$ & $\begin{array}{c}0.0000 \\
(0.00) \\
\end{array}$ & $\begin{array}{l}0.0000 \\
(0.00)\end{array}$ & $\begin{array}{c}0.0000 \\
(0.00)\end{array}$ & $\begin{array}{l}0.0000 \\
(0.00)\end{array}$ & $\begin{array}{l}0.0000 \\
(0.00)\end{array}$ & $\begin{array}{l}0.0000 \\
(0.00)\end{array}$ & & $\begin{array}{l}0.0000 \\
(0.00)\end{array}$ & $\begin{array}{l}0.0000 \\
(0.00)\end{array}$ & $\begin{array}{l}0.0000 \\
(0.00)\end{array}$ & $\begin{array}{l}0.0000 \\
(0.00)\end{array}$ & $\begin{array}{c}0.0000 \\
(0.00) \\
\end{array}$ & 283.83 \\
\hline USA & $\begin{array}{l}0.0000 \\
(0.00)\end{array}$ & $\begin{array}{c}0.1997 \\
(429.60) \\
\end{array}$ & $\begin{array}{l}0.0000 \\
(0.00)\end{array}$ & $\begin{array}{l}0.0000 \\
(0.00) \\
\end{array}$ & $\begin{array}{c}0.0000 \\
(0.00) \\
\end{array}$ & $\begin{array}{c}0.0000 \\
(0.00)\end{array}$ & $\begin{array}{c}0.2364 \\
(508.55) \\
\end{array}$ & \begin{tabular}{|c|}
0.2138 \\
$(459.93)$ \\
\end{tabular} & $\begin{array}{l}0.0000 \\
(0.00) \\
\end{array}$ & \begin{tabular}{|l|}
0.1234 \\
$(265.5)$ \\
\end{tabular} & $\begin{array}{l}0.0000 \\
(0.00) \\
\end{array}$ & $\begin{array}{c}0.0000 \\
(0.00)\end{array}$ & $\begin{array}{l}0.0000 \\
(0.00)\end{array}$ & $\begin{array}{l}0.0000 \\
(0.00)\end{array}$ & $\begin{array}{l}0.0000 \\
(0.00)\end{array}$ & & $\begin{array}{l}0.0000 \\
(0.00) \\
\end{array}$ & $\begin{array}{l}0.0000 \\
(0.00)\end{array}$ & $\begin{array}{l}0.0000 \\
(0.00)\end{array}$ & $\begin{array}{l}0.0000 \\
(0.00) \\
\end{array}$ & $\begin{array}{c}0.2267 \\
(487.68) \\
\end{array}$ & 2151.22 \\
\hline Uzbekistan & $\begin{array}{l}0.0000 \\
(0.00)\end{array}$ & $\begin{array}{c}0.7224 \\
(152.47)\end{array}$ & $\begin{array}{l}0.0000 \\
(0.00)\end{array}$ & $\begin{array}{c}0.0000 \\
(0.00)\end{array}$ & $\begin{array}{c}0.0000 \\
(0.00)\end{array}$ & $\begin{array}{l}0.0000 \\
(0.00)\end{array}$ & $\begin{array}{c}0.0000 \\
(0.00)\end{array}$ & $\begin{array}{l}0.0000 \\
(0.00)\end{array}$ & $\begin{array}{l}0.0000 \\
(0.00)\end{array}$ & \begin{tabular}{|l|}
0.2776 \\
$(59.59)$
\end{tabular} & $\begin{array}{c}0.0000 \\
(0.00)\end{array}$ & $\begin{array}{c}0.0000 \\
(0.00)\end{array}$ & $\begin{array}{c}0.0000 \\
(0.00)\end{array}$ & $\begin{array}{c}0.0000 \\
(0.00)\end{array}$ & $\begin{array}{l}0.0000 \\
(0.00)\end{array}$ & $\begin{array}{c}0.0000 \\
(0.00)\end{array}$ & $\begin{array}{l}0.0000 \\
(0.00)\end{array}$ & $\begin{array}{c}0.0000 \\
(0.00)\end{array}$ & $\begin{array}{c}0.0000 \\
(0.00)\end{array}$ & $\begin{array}{c}0.0000 \\
(0.00)\end{array}$ & $\begin{array}{c}0.0000 \\
(0.00)\end{array}$ & 211.06 \\
\hline Cameroon & $\begin{array}{c}0.0000 \\
(0.00)\end{array}$ & $\begin{array}{c}0.0000 \\
(0.00)\end{array}$ & $\begin{array}{l}0.0000 \\
(0.00) \\
\end{array}$ & $\begin{array}{c}0.0000 \\
(0.00) \\
\end{array}$ & $\begin{array}{c}0.0000 \\
(0.00) \\
\end{array}$ & $\begin{array}{c}0.0000 \\
(0.00) \\
\end{array}$ & $\begin{array}{c}0.0000 \\
(0.00) \\
\end{array}$ & \begin{tabular}{|l|}
1.0000 \\
$(76.89)$ \\
\end{tabular} & $\begin{array}{c}0.0000 \\
(0.00) \\
\end{array}$ & \begin{tabular}{|c|}
0.0000 \\
$(0.00)$ \\
\end{tabular} & $\begin{array}{c}0.0000 \\
(0.00) \\
\end{array}$ & $\begin{array}{c}0.0000 \\
(0.00)\end{array}$ & $\begin{array}{c}0.0000 \\
(0.00)\end{array}$ & $\begin{array}{c}0.0000 \\
(0.00) \\
\end{array}$ & $\begin{array}{c}0.0000 \\
(0.00)\end{array}$ & $\begin{array}{c}0.0000 \\
(0.00) \\
\end{array}$ & $\begin{array}{l}0.0000 \\
(0.00) \\
\end{array}$ & $\begin{array}{c}0.0000 \\
(0.00)\end{array}$ & $\begin{array}{c}0.0000 \\
(0.00)\end{array}$ & $\begin{array}{c}0.0000 \\
(0.00)\end{array}$ & $\begin{array}{c}0.0000 \\
(0.00) \\
\end{array}$ & 76.89 \\
\hline China & $\begin{array}{c}0.0000 \\
(0.00)\end{array}$ & $\begin{array}{c}0.0000 \\
(0.00)\end{array}$ & $\begin{array}{l}0.0000 \\
(0.00)\end{array}$ & $\begin{array}{c}0.0000 \\
(0.00)\end{array}$ & $\begin{array}{c}0.0000 \\
(0.00)\end{array}$ & $\begin{array}{l}0.0000 \\
(0.00)\end{array}$ & $\begin{array}{c}0.0000 \\
(0.00)\end{array}$ & $\begin{array}{c}1.0000 \\
(171.78)\end{array}$ & $\begin{array}{l}0.0000 \\
(0.00)\end{array}$ & \begin{tabular}{|c|}
0.0000 \\
$(0.00)$
\end{tabular} & $\begin{array}{c}0.0000 \\
(0.00)\end{array}$ & $\begin{array}{c}0.0000 \\
(0.00)\end{array}$ & $\begin{array}{c}\mathbf{0 . 0 0 0 0} \\
(\mathbf{0 . 0 0})\end{array}$ & $\begin{array}{c}0.0000 \\
(0.00)\end{array}$ & $\begin{array}{c}0.0000 \\
(0.00)\end{array}$ & $\begin{array}{c}0.0000 \\
(0.00)\end{array}$ & $\begin{array}{l}0.0000 \\
(0.00)\end{array}$ & $\begin{array}{c}0.0000 \\
(0.00)\end{array}$ & $\begin{array}{c}0.0000 \\
(0.00)\end{array}$ & $\begin{array}{c}0.0000 \\
(0.00)\end{array}$ & $\begin{array}{l}0.0000 \\
(0.00)\end{array}$ & 171.78 \\
\hline Israel & $\begin{array}{c}0.0000 \\
(0.00)\end{array}$ & $\begin{array}{c}0.0000 \\
(0.00)\end{array}$ & $\begin{array}{c}0.0000 \\
(0.00)\end{array}$ & $\begin{array}{c}0.0000 \\
(0.00)\end{array}$ & $\begin{array}{c}0.0000 \\
(0.00)\end{array}$ & $\begin{array}{l}0.0000 \\
(0.00)\end{array}$ & $\begin{array}{l}1.0000 \\
(84.44)\end{array}$ & $\begin{array}{c}0.0000 \\
(0.00)\end{array}$ & $\begin{array}{l}0.0000 \\
(0.00)\end{array}$ & \begin{tabular}{|c|}
0.0000 \\
$(0.00)$
\end{tabular} & $\begin{array}{c}0.0000 \\
(0.00)\end{array}$ & $\begin{array}{c}0.0000 \\
(0.00)\end{array}$ & $\begin{array}{c}0.0000 \\
(0.00)\end{array}$ & $\begin{array}{c}\mathbf{0 . 0 0 0 0} \\
(0.00)\end{array}$ & $\begin{array}{c}0.0000 \\
(0.00)\end{array}$ & $\begin{array}{c}0.0000 \\
(0.00)\end{array}$ & $\begin{array}{l}0.0000 \\
(0.00)\end{array}$ & $\begin{array}{c}0.0000 \\
(0.00)\end{array}$ & $\begin{array}{l}0.0000 \\
(0.00)\end{array}$ & $\begin{array}{c}0.0000 \\
(0.00)\end{array}$ & $\begin{array}{l}0.0000 \\
(0.00)\end{array}$ & 84.44 \\
\hline Malaysia & $\begin{array}{c}0.0000 \\
(0.00)\end{array}$ & $\begin{array}{c}0.0000 \\
(0.00)\end{array}$ & $\begin{array}{l}0.0000 \\
(0.00)\end{array}$ & $\begin{array}{c}0.0000 \\
(0.00)\end{array}$ & $\begin{array}{c}0.0000 \\
(0.00)\end{array}$ & $\begin{array}{l}0.0000 \\
(0.00)\end{array}$ & $\begin{array}{l}0.0000 \\
(0.00)\end{array}$ & \begin{tabular}{|l|}
1.0000 \\
$(13.11)$
\end{tabular} & $\begin{array}{l}0.0000 \\
(0.00)\end{array}$ & \begin{tabular}{|c|}
0.0000 \\
$(0.00)$
\end{tabular} & $\begin{array}{c}0.0000 \\
(0.00)\end{array}$ & $\begin{array}{c}0.0000 \\
(0.00)\end{array}$ & $\begin{array}{c}0.0000 \\
(0.00)\end{array}$ & $\begin{array}{c}0.0000 \\
(0.00)\end{array}$ & $\begin{array}{c}.0000 \\
(0.00)\end{array}$ & $\begin{array}{c}0.0000 \\
(0.00)\end{array}$ & $\begin{array}{l}0.0000 \\
(0.00)\end{array}$ & $\begin{array}{c}0.0000 \\
(0.00)\end{array}$ & $\begin{array}{l}0.0000 \\
(0.00)\end{array}$ & $\begin{array}{c}0.0000 \\
(0.00)\end{array}$ & $\begin{array}{l}0.0000 \\
(0.00)\end{array}$ & 13.11 \\
\hline \begin{tabular}{|l|} 
Russian \\
Federation \\
\end{tabular} & $\begin{array}{l}0.0000 \\
(0.00)\end{array}$ & $\begin{array}{l}0.0000 \\
(0.00)\end{array}$ & $\begin{array}{l}0.0000 \\
(0.00)\end{array}$ & $\begin{array}{l}0.0000 \\
(0.00)\end{array}$ & $\begin{array}{l}0.0000 \\
(0.00)\end{array}$ & $\begin{array}{l}0.0000 \\
(0.00)\end{array}$ & $\begin{array}{l}0.0000 \\
(0.00)\end{array}$ & \begin{tabular}{|c|}
1.0000 \\
$(110.17)$ \\
\end{tabular} & $\begin{array}{l}0.0000 \\
(0.00)\end{array}$ & \begin{tabular}{|l|}
0.0000 \\
$(0.00)$ \\
\end{tabular} & $\begin{array}{l}0.0000 \\
(0.00)\end{array}$ & $\begin{array}{l}0.0000 \\
(0.00)\end{array}$ & $\begin{array}{l}0.0000 \\
(0.00)\end{array}$ & $\begin{array}{l}0.0000 \\
(0.00)\end{array}$ & $\begin{array}{l}0.0000 \\
(0.00)\end{array}$ & $\begin{array}{l}.0000 \\
(0.00)\end{array}$ & $\begin{array}{l}0.0000 \\
(0.00)\end{array}$ & $\begin{array}{l}0.0000 \\
(0.00)\end{array}$ & $\begin{array}{l}0.0000 \\
(0.00)\end{array}$ & $\begin{array}{l}0.0000 \\
(0.00)\end{array}$ & $\begin{array}{l}0.0000 \\
(0.00)\end{array}$ & 110.17 \\
\hline Switzerland & $\begin{array}{l}0.0000 \\
(0.00)\end{array}$ & $\begin{array}{l}0.0000 \\
(0.00)\end{array}$ & $\begin{array}{l}0.0000 \\
(0.00)\end{array}$ & $\begin{array}{l}0.0000 \\
(0.00)\end{array}$ & $\begin{array}{l}0.0000 \\
(0.00)\end{array}$ & $\begin{array}{c}0.0000 \\
(0.00)\end{array}$ & $\begin{array}{l}0.0000 \\
(0.00)\end{array}$ & \begin{tabular}{|c|}
1.0000 \\
$(191.44)$ \\
\end{tabular} & $\begin{array}{l}0.0000 \\
(0.00)\end{array}$ & \begin{tabular}{|c|}
0.0000 \\
$(0.00)$
\end{tabular} & $\begin{array}{l}0.0000 \\
(0.00)\end{array}$ & $\begin{array}{c}0.0000 \\
(0.00)\end{array}$ & $\begin{array}{l}0.0000 \\
(0.00)\end{array}$ & $\begin{array}{l}0.0000 \\
(0.00)\end{array}$ & $\begin{array}{l}0.0000 \\
(0.00)\end{array}$ & $\begin{array}{l}0.0000 \\
(0.00)\end{array}$ & $\begin{array}{l}\mathbf{0 . 0 0 0 0} \\
(\mathbf{0 . 0 0 )}\end{array}$ & $\begin{array}{l}0.0000 \\
(0.00)\end{array}$ & $\begin{array}{l}0.0000 \\
(0.00)\end{array}$ & $\begin{array}{l}0.0000 \\
(0.00)\end{array}$ & $\begin{array}{l}0.0000 \\
(0.00)\end{array}$ & 191.44 \\
\hline Turkey & $\begin{array}{c}0.0000 \\
(0.00)\end{array}$ & $\begin{array}{c}0.0000 \\
(0.00)\end{array}$ & $\begin{array}{c}0.0000 \\
(0.00)\end{array}$ & $\begin{array}{c}0.0000 \\
(0.00)\end{array}$ & $\begin{array}{c}0.0000 \\
(0.00)\end{array}$ & $\begin{array}{l}0.0000 \\
(0.00)\end{array}$ & $\begin{array}{c}0.0000 \\
(0.00)\end{array}$ & \begin{tabular}{|c|}
0.0000 \\
$(0.00)$
\end{tabular} & $\begin{array}{c}0.0000 \\
(0.00)\end{array}$ & \begin{tabular}{|c|}
0.0000 \\
$(0.00)$
\end{tabular} & $\begin{array}{c}0.0000 \\
(0.00)\end{array}$ & $\begin{array}{c}0.0000 \\
(0.00)\end{array}$ & $\begin{array}{c}0.0000 \\
(0.00)\end{array}$ & $\begin{array}{c}0.0000 \\
(0.00)\end{array}$ & $\begin{array}{c}0.0000 \\
(0.00)\end{array}$ & $\begin{array}{c}1.0000 \\
(223.22)\end{array}$ & $\begin{array}{l}0.0000 \\
(0.00)\end{array}$ & $\begin{array}{c}\mathbf{0 . 0 0 0 0} \\
(\mathbf{0 . 0 0})\end{array}$ & $\begin{array}{l}0.0000 \\
(0.00)\end{array}$ & $\begin{array}{c}0.0000 \\
(0.00)\end{array}$ & $\begin{array}{c}0.0000 \\
(0.00)\end{array}$ & 223.22 \\
\hline Turkmenistan & $\begin{array}{c}0.0000 \\
(0.00)\end{array}$ & $\begin{array}{c}0.0000 \\
(0.00)\end{array}$ & $\begin{array}{l}0.0000 \\
(0.00)\end{array}$ & $\begin{array}{c}0.0000 \\
(0.00)\end{array}$ & $\begin{array}{c}0.0000 \\
(0.00)\end{array}$ & $\begin{array}{l}0.0000 \\
(0.00)\end{array}$ & $\begin{array}{c}0.0000 \\
(0.00)\end{array}$ & $\begin{array}{l}0.0000 \\
(0.00)\end{array}$ & $\begin{array}{c}0.0000 \\
(0.00)\end{array}$ & \begin{tabular}{|c|}
0.0000 \\
$(0.00)$
\end{tabular} & $\begin{array}{c}0.0000 \\
(0.00)\end{array}$ & $\begin{array}{c}0.8171 \\
(109.31)\end{array}$ & $\begin{array}{c}0.0000 \\
(0.00)\end{array}$ & $\begin{array}{c}0.0000 \\
(0.00)\end{array}$ & $\begin{array}{l}0.1829 \\
(24.46)\end{array}$ & $\begin{array}{l}0.0000 \\
(0.00)\end{array}$ & $\begin{array}{l}0.0000 \\
(0.00)\end{array}$ & $\begin{array}{c}0.0000 \\
(0.00)\end{array}$ & $\begin{array}{l}0.0000 \\
(0.00)\end{array}$ & $\begin{array}{c}0.0000 \\
(0.00)\end{array}$ & $\begin{array}{c}0.0000 \\
(0.00)\end{array}$ & 133.78 \\
\hline Austria & $\begin{array}{c}0.0000 \\
(0.00)\end{array}$ & $\begin{array}{c}0.0000 \\
(0.00)\end{array}$ & $\begin{array}{l}0.0000 \\
(0.00)\end{array}$ & $\begin{array}{c}0.0000 \\
(0.00)\end{array}$ & $\begin{array}{c}0.0000 \\
(0.00)\end{array}$ & $\begin{array}{l}0.0000 \\
(0.00)\end{array}$ & $\begin{array}{c}0.0000 \\
(0.00)\end{array}$ & $\begin{array}{l}1.0000 \\
(4.39)\end{array}$ & $\begin{array}{c}0.0000 \\
(0.00)\end{array}$ & \begin{tabular}{|c|}
0.0000 \\
$(0.00)$
\end{tabular} & $\begin{array}{c}0.0000 \\
(0.00)\end{array}$ & $\begin{array}{c}0.0000 \\
(0.00)\end{array}$ & $\begin{array}{c}0.0000 \\
(0.00)\end{array}$ & $\begin{array}{c}0.0000 \\
(0.00)\end{array}$ & $\begin{array}{c}0.0000 \\
(0.00)\end{array}$ & $\begin{array}{c}0.0000 \\
(0.00)\end{array}$ & $\begin{array}{l}0.0000 \\
(0.00)\end{array}$ & $\begin{array}{c}0.0000 \\
(0.00)\end{array}$ & $\begin{array}{l}0.0000 \\
(0.00)\end{array}$ & $\begin{array}{c}\mathbf{0 . 0 0 0 0} \\
(\mathbf{0 . 0 0})\end{array}$ & $\begin{array}{c}0.0000 \\
(0.00)\end{array}$ & 4.39 \\
\hline Other & $\begin{array}{c}0.0000 \\
(0.00) \\
\end{array}$ & $\begin{array}{c}0.0000 \\
(0.00) \\
\end{array}$ & $\begin{array}{l}0.0000 \\
(0.00) \\
\end{array}$ & $\begin{array}{c}0.0000 \\
(0.00) \\
\end{array}$ & $\begin{array}{c}0.0000 \\
(0.00) \\
\end{array}$ & $\begin{array}{l}0.0000 \\
(0.00) \\
\end{array}$ & $\begin{array}{c}1.0000 \\
(1244.44) \\
\end{array}$ & $\begin{array}{l}0.0000 \\
(0.00) \\
\end{array}$ & $\begin{array}{c}0.0000 \\
(0.00) \\
\end{array}$ & \begin{tabular}{|c}
0.0000 \\
$(0.00)$ \\
\end{tabular} & $\begin{array}{c}0.0000 \\
(0.00) \\
\end{array}$ & $\begin{array}{c}0.0000 \\
(0.00) \\
\end{array}$ & $\begin{array}{c}0.0000 \\
(0.00) \\
\end{array}$ & $\begin{array}{c}0.0000 \\
(0.00) \\
\end{array}$ & $\begin{array}{c}0.0000 \\
(0.00) \\
\end{array}$ & $\begin{array}{l}0.0000 \\
(0.00) \\
\end{array}$ & $\begin{array}{c}0.0000 \\
(0.00) \\
\end{array}$ & $\begin{array}{c}0.0000 \\
(0.00) \\
\end{array}$ & $\begin{array}{c}0.0000 \\
(0.00) \\
\end{array}$ & $\begin{array}{l}0.0000 \\
(0.00) \\
\end{array}$ & $\begin{array}{c}\mathbf{0 . 0 0 0 0} \\
(\mathbf{0 . 0 0}) \\
\end{array}$ & 1244.44 \\
\hline Total Import & 1894.16 & 1164.14 & 212.12 & 1602.73 & 0.00 & 37.27 & 3885.93 & 4021.81 & 117.13 & 2385.14 & 0.00 & 213.11 & 263.70 & 103.51 & 43.71 & 367.68 & 366.45 & 265.88 & 188.37 & 0.00 & 2546.36 & \\
\hline Percentage & 83.45 & 100.58 & 38.96 & 197.71 & 0.00 & 6.66 & 71.87 & 181.90 & 41.27 & 110.87 & 0.00 & 277.17 & 153.51 & 122.58 & 333.38 & 333.75 & 191.41 & 119.11 & 140.81 & 0.00 & 204.62 & \\
\hline $\begin{array}{l}\% \quad \text { Increase/ } \\
\text { Decrease }\end{array}$ & -16.55 & 0.58 & -61.04 & 97.71 & -100.00 & -93.34 & -28.13 & 81.90 & -58.73 & 10.87 & -100.00 & 177.17 & 53.51 & 22.58 & 233.38 & 233.75 & 91.41 & 19.11 & 40.81 & -100.00 & 104.62 & \\
\hline
\end{tabular}

Note: Figures in the parenthesis indicate average quantity of import (in tons) by India 
Table.6 Transitional probability matrix of cotton imports in the post-WTO period (1995-96 to 2012-13)

\begin{tabular}{|c|c|c|c|c|c|c|c|c|c|c|c|c|c|c|c|c|c|c|c|c|c|c|}
\hline & Australia & Benin & $\begin{array}{c}\text { Burkina } \\
\text { Faso }\end{array}$ & $\begin{array}{c}\text { Cote } \\
\text { delvoire }\end{array}$ & Egypt & Mali & Sudan & Tanzania & USA & Uzbekistan & Argentina & Cameroon & China & Greece & Israel & Malaysia & Nigeria & $\begin{array}{c}\text { Russian } \\
\text { Federation }\end{array}$ & $\begin{array}{c}\text { South } \\
\text { Africa } \\
\end{array}$ & Switzerland & Others & \begin{tabular}{|c|} 
Average \\
Import
\end{tabular} \\
\hline Australia & $\begin{array}{c}\mathbf{0 . 0 4 1 2} \\
(337.16) \\
\end{array}$ & \begin{tabular}{|c|}
0.1387 \\
$(1134.97)$ \\
\end{tabular} & $\begin{array}{c}0.0590 \\
(482.28) \\
\end{array}$ & $\begin{array}{c}0.1111 \\
(908.63) \\
\end{array}$ & $\begin{array}{c}0.0000 \\
(0.00) \\
\end{array}$ & $\begin{array}{c}0.0289 \\
(236.43) \\
\end{array}$ & $\begin{array}{c}0.0000 \\
(0.00) \\
\end{array}$ & $\begin{array}{c}0.0000 \\
(0.00)\end{array}$ & $\begin{array}{c}0.0000 \\
(0.00) \\
\end{array}$ & $\begin{array}{c}0.0258 \\
(210.87) \\
\end{array}$ & $\begin{array}{c}0.0173 \\
(141.25) \\
\end{array}$ & $\begin{array}{c}0.0000 \\
(0.00)\end{array}$ & $\begin{array}{c}0.0612 \\
(501.02) \\
\end{array}$ & $\begin{array}{c}0.0161 \\
(132.05) \\
\end{array}$ & $\begin{array}{l}0.0000 \\
(0.00)\end{array}$ & $\begin{array}{c}0.0000 \\
(0.00)\end{array}$ & $\begin{array}{c}0.0000 \\
(0.00) \\
\end{array}$ & $\begin{array}{c}0.0781 \\
(638.72) \\
\end{array}$ & $\begin{array}{c}0.0776 \\
(634.91) \\
\end{array}$ & $\begin{array}{c}0.0194 \\
(158.87) \\
\end{array}$ & \begin{tabular}{|c|}
0.3256 \\
$(2663.32)$ \\
\end{tabular} & 8180.50 \\
\hline Benin & $\begin{array}{l}0.0000 \\
(0.00)\end{array}$ & \begin{tabular}{|l|}
0.0000 \\
$(0.00)$
\end{tabular} & $\begin{array}{l}0.0000 \\
(0.00)\end{array}$ & $\begin{array}{c}0.0730 \\
(440.87)\end{array}$ & $\begin{array}{l}0.0000 \\
(0.00)\end{array}$ & $\begin{array}{c}0.0802 \\
(484.85)\end{array}$ & $\begin{array}{l}0.0000 \\
(0.00)\end{array}$ & $\begin{array}{l}0.0000 \\
(0.00)\end{array}$ & $\begin{array}{l}0.0000 \\
(0.00)\end{array}$ & $\begin{array}{c}0.0338 \\
(204.18)\end{array}$ & $\begin{array}{l}0.0000 \\
(0.00)\end{array}$ & $\begin{array}{c}0.0194 \\
(117.42)\end{array}$ & \begin{tabular}{|c|}
0.0000 \\
$(0.00)$
\end{tabular} & $\begin{array}{c}0.1417 \\
(856.43) \\
\end{array}$ & $\begin{array}{l}0.0000 \\
(0.00)\end{array}$ & $\begin{array}{l}0.0000 \\
(0.00)\end{array}$ & $\begin{array}{l}0.0000 \\
(0.00)\end{array}$ & $\begin{array}{l}0.0000 \\
(0.00)\end{array}$ & $\begin{array}{l}0.0000 \\
(0.00)\end{array}$ & $\begin{array}{l}0.0000 \\
(0.00)\end{array}$ & \begin{tabular}{|c|}
0.6519 \\
$(3939.30)$
\end{tabular} & 6043.06 \\
\hline \begin{tabular}{|l|} 
Burkina \\
Faso
\end{tabular} & $\begin{array}{l}0.0000 \\
(0.00)\end{array}$ & $\begin{array}{l}0.0000 \\
(0.00)\end{array}$ & $\begin{array}{c}0.0480 \\
(213.09)\end{array}$ & $\begin{array}{l}0.0000 \\
(0.00)\end{array}$ & $\begin{array}{l}0.0000 \\
(0.00)\end{array}$ & $\begin{array}{l}0.0000 \\
(0.00)\end{array}$ & $\begin{array}{l}0.0000 \\
(0.00)\end{array}$ & $\begin{array}{c}0.9073 \\
(4028.19) \\
\end{array}$ & $\begin{array}{l}0.0000 \\
(0.00)\end{array}$ & $\begin{array}{l}0.0000 \\
(0.00)\end{array}$ & $\begin{array}{l}0.0000 \\
(0.00)\end{array}$ & $\begin{array}{l}0.0000 \\
(0.00)\end{array}$ & \begin{tabular}{|c|}
0.0000 \\
$(0.00)$
\end{tabular} & \begin{tabular}{|c|}
0.0447 \\
$(198.44)$ \\
\end{tabular} & $\begin{array}{l}0.0000 \\
(0.00)\end{array}$ & $\begin{array}{l}0.0000 \\
(0.00)\end{array}$ & $\begin{array}{c}0.0000 \\
(0.00)\end{array}$ & $\begin{array}{l}0.0000 \\
(0.00)\end{array}$ & $\begin{array}{l}0.0000 \\
(0.00)\end{array}$ & $\begin{array}{l}0.0000 \\
(0.00)\end{array}$ & $\begin{array}{c}0.0000 \\
(0.00) \\
\end{array}$ & 4439.72 \\
\hline \begin{tabular}{|l|}
$\begin{array}{l}\text { Cote } \\
\text { deIvoire }\end{array}$ \\
\end{tabular} & $\begin{array}{c}0.3938 \\
(202.99) \\
\end{array}$ & $\begin{array}{l}0.0000 \\
(0.00)\end{array}$ & $\begin{array}{c}0.0000 \\
(0.00)\end{array}$ & $\begin{array}{l}.0000 \\
(0.00)\end{array}$ & $\begin{array}{l}0.0000 \\
(0.00)\end{array}$ & $\begin{array}{l}0.0000 \\
(0.00)\end{array}$ & $\begin{array}{c}0.0000 \\
(0.00)\end{array}$ & $\begin{array}{c}0.0000 \\
(0.00) \\
\end{array}$ & $\begin{array}{c}0.4493 \\
(2296.70) \\
\end{array}$ & $\begin{array}{c}0.0000 \\
(0.00)\end{array}$ & $\begin{array}{l}0.0000 \\
(0.00)\end{array}$ & $\begin{array}{l}0.0000 \\
(0.00)\end{array}$ & \begin{tabular}{|c|}
0.0000 \\
$(0.00)$ \\
\end{tabular} & $\begin{array}{c}0.0000 \\
(0.00) \\
\end{array}$ & $\begin{array}{c}0.0000 \\
(0.00)\end{array}$ & $\begin{array}{l}0.0000 \\
(0.00)\end{array}$ & $\begin{array}{c}0.1568 \\
(801.70) \\
\end{array}$ & $\begin{array}{c}0.0000 \\
(0.00)\end{array}$ & $\begin{array}{c}.0000 \\
(0.00)\end{array}$ & $\begin{array}{l}0.0000 \\
(0.00)\end{array}$ & $\begin{array}{l}0.0000 \\
(0.00)\end{array}$ & 5111.39 \\
\hline Egypt & $\begin{array}{c}0.1237 \\
(2030.28)\end{array}$ & $\begin{array}{c}0.0000 \\
(0.00)\end{array}$ & $\begin{array}{l}0.0000 \\
(0.00)\end{array}$ & $\begin{array}{c}0.0000 \\
(0.00)\end{array}$ & \begin{tabular}{|c|}
$\mathbf{0 . 2 4 3 1}$ \\
$(3988.98)$
\end{tabular} & $\begin{array}{c}0.0000 \\
(0.00)\end{array}$ & \begin{tabular}{|c|}
0.1360 \\
$(2231.34)$
\end{tabular} & $\begin{array}{c}0.0000 \\
(0.00)\end{array}$ & $\begin{array}{c}0.4349 \\
(7136.37)\end{array}$ & $\begin{array}{c}0.0000 \\
(0.00)\end{array}$ & $\begin{array}{c}0.0000 \\
(0.00)\end{array}$ & $\begin{array}{c}0.0000 \\
(0.00)\end{array}$ & \begin{tabular}{|c|}
0.0000 \\
$(0.00)$
\end{tabular} & $\begin{array}{c}0.0000 \\
(0.00)\end{array}$ & \begin{tabular}{|c|}
0.0346 \\
$(568.44)$
\end{tabular} & $\begin{array}{c}0.0000 \\
(0.00)\end{array}$ & $\begin{array}{l}0.0000 \\
(0.00)\end{array}$ & $\begin{array}{c}0.0000 \\
(0.00)\end{array}$ & $\begin{array}{c}0.0000 \\
(0.00)\end{array}$ & $\begin{array}{l}0.0054 \\
(89.33)\end{array}$ & $\begin{array}{c}0.0221 \\
(363.09)\end{array}$ & 16407.83 \\
\hline Mali & $\begin{array}{l}0.0000 \\
(0.00)\end{array}$ & $\begin{array}{c}0.2062 \\
(910.06)\end{array}$ & $\begin{array}{c}0.0000 \\
(0.00)\end{array}$ & $\begin{array}{c}0.0000 \\
(0.00)\end{array}$ & $\begin{array}{l}0.0000 \\
(0.00)\end{array}$ & \begin{tabular}{|c|}
0.2716 \\
$(1198.59)$
\end{tabular} & \begin{tabular}{|c|}
0.0000 \\
$(0.00)$
\end{tabular} & $\begin{array}{c}0.0000 \\
(0.00)\end{array}$ & \begin{tabular}{|c|}
0.0000 \\
$(0.00)$
\end{tabular} & $\begin{array}{c}0.0000 \\
(0.00)\end{array}$ & $\begin{array}{c}0.0000 \\
(0.00)\end{array}$ & $\begin{array}{c}0.1895 \\
(836.43)\end{array}$ & \begin{tabular}{|c|}
0.0000 \\
$(0.00)$
\end{tabular} & \begin{tabular}{|c|}
0.0927 \\
$(409.10)$ \\
\end{tabular} & $\begin{array}{l}0.0000 \\
(0.00)\end{array}$ & $\begin{array}{c}0.0000 \\
(0.00)\end{array}$ & $\begin{array}{c}0.0000 \\
(0.00)\end{array}$ & $\begin{array}{l}0.0000 \\
(0.00)\end{array}$ & $\begin{array}{c}0.0000 \\
(0.00)\end{array}$ & $\begin{array}{c}0.0000 \\
(0.00)\end{array}$ & $\begin{array}{c}0.2401 \\
(1059.55)\end{array}$ & 4413.72 \\
\hline Sudan & $\begin{array}{l}0.0000 \\
(0.00)\end{array}$ & \begin{tabular}{|c|}
0.0000 \\
$(0.00)$
\end{tabular} & $\begin{array}{l}0.0000 \\
(0.00)\end{array}$ & $\begin{array}{l}0.0000 \\
(0.00)\end{array}$ & $\begin{array}{l}0.0000 \\
(0.00)\end{array}$ & $\begin{array}{c}0.0000 \\
(0.00) \\
\end{array}$ & $\begin{array}{l}.0000 \\
(0.00)\end{array}$ & $\begin{array}{l}0.0170 \\
(58.90)\end{array}$ & $\begin{array}{c}0.0000 \\
(0.00)\end{array}$ & $\begin{array}{l}0.0000 \\
(0.00)\end{array}$ & $\begin{array}{l}0.0000 \\
(0.00)\end{array}$ & $\begin{array}{c}0.0693 \\
(240.00)\end{array}$ & $\begin{array}{c}0.0000 \\
(0.00)\end{array}$ & $\begin{array}{l}0.0000 \\
(0.00) \\
\end{array}$ & $\begin{array}{l}0.0000 \\
(0.00)\end{array}$ & $\begin{array}{l}0.0000 \\
(0.00)\end{array}$ & $\begin{array}{c}0.0000 \\
(0.00)\end{array}$ & $\begin{array}{l}0.0000 \\
(0.00)\end{array}$ & $\begin{array}{l}0.0000 \\
(0.00)\end{array}$ & $\begin{array}{l}0.0000 \\
(0.00)\end{array}$ & \begin{tabular}{|c|}
0.9137 \\
$(3166.27)$ \\
\end{tabular} & 3465.17 \\
\hline Tanzania & $\begin{array}{c}0.0000 \\
(0.00)\end{array}$ & $\begin{array}{l}0.0000 \\
(0.00)\end{array}$ & $\begin{array}{c}0.0000 \\
(0.00)\end{array}$ & $\begin{array}{l}0.0000 \\
(0.00)\end{array}$ & \begin{tabular}{|c|}
0.0390 \\
$(232.38)$
\end{tabular} & $\begin{array}{c}0.0000 \\
(0.00)\end{array}$ & \begin{tabular}{|c|}
0.2824 \\
$(1681.65)$
\end{tabular} & $\begin{array}{l}\mathbf{0 . 0 0 7 0} \\
(41.50)\end{array}$ & \begin{tabular}{|c|}
0.2725 \\
$(1622.92)$
\end{tabular} & $\begin{array}{c}0.0000 \\
(0.00)\end{array}$ & $\begin{array}{c}0.0000 \\
(0.00)\end{array}$ & $\begin{array}{c}0.0000 \\
(0.00)\end{array}$ & \begin{tabular}{|c|}
0.0000 \\
$(0.00)$
\end{tabular} & $\begin{array}{c}0.0000 \\
(0.00)\end{array}$ & $\begin{array}{c}0.1469 \\
(875.08)\end{array}$ & $\begin{array}{l}0.0012 \\
(7.15)\end{array}$ & $\begin{array}{c}0.0249 \\
(148.37)\end{array}$ & $\begin{array}{c}.0000 \\
(0.00)\end{array}$ & $\begin{array}{c}0.0000 \\
(0.00)\end{array}$ & $\begin{array}{c}0.0000 \\
(0.00)\end{array}$ & $\begin{array}{c}0.2261 \\
(1346.83)\end{array}$ & 5955.89 \\
\hline USA & $\begin{array}{c}0.0000 \\
(0.00)\end{array}$ & $\begin{array}{c}0.0000 \\
(0.00)\end{array}$ & $\begin{array}{c}0.0164 \\
(579.03)\end{array}$ & $\begin{array}{c}0.0000 \\
(0.00)\end{array}$ & $\begin{array}{c}0.4428 \\
(15666.6)\end{array}$ & $\begin{array}{c}0.0000 \\
(0.00)\end{array}$ & $\begin{array}{c}0.0000 \\
(0.00)\end{array}$ & $\begin{array}{c}0.0000 \\
(0.00)\end{array}$ & \begin{tabular}{|c|}
0.4606 \\
$(16298.2)$
\end{tabular} & $\begin{array}{c}0.0000 \\
(0.00)\end{array}$ & $\begin{array}{c}0.0000 \\
(0.00)\end{array}$ & $\begin{array}{c}0.0000 \\
(0.00)\end{array}$ & \begin{tabular}{|l|}
0.0000 \\
$(0.00)$
\end{tabular} & $\begin{array}{l}0.0000 \\
(0.00)\end{array}$ & $\begin{array}{l}0.0000 \\
(0.00)\end{array}$ & $\begin{array}{c}0.0000 \\
(0.00)\end{array}$ & $\begin{array}{l}0.0027 \\
(96.83)\end{array}$ & $\begin{array}{l}0.0000 \\
(0.00)\end{array}$ & $\begin{array}{c}0.0000 \\
(0.00)\end{array}$ & $\begin{array}{c}0.0000 \\
(0.00)\end{array}$ & & 35381.94 \\
\hline Uzbekistan & $\begin{array}{c}0.0000 \\
(0.00)\end{array}$ & $\begin{array}{c}0.0000 \\
(0.00)\end{array}$ & $\begin{array}{l}0.0000 \\
(0.00)\end{array}$ & $\begin{array}{c}0.0000 \\
(0.00)\end{array}$ & $\begin{array}{c}0.0000 \\
(0.00)\end{array}$ & $\begin{array}{c}0.0000 \\
(0.00)\end{array}$ & $\begin{array}{c}0.0000 \\
(0.00)\end{array}$ & $\begin{array}{c}0.0000 \\
(0.00)\end{array}$ & $\begin{array}{c}1.0000 \\
(4768.17)\end{array}$ & $\begin{array}{c}0.0000 \\
(0.00)\end{array}$ & $\begin{array}{c}0.0000 \\
(0.00)\end{array}$ & $\begin{array}{c}0.0000 \\
(0.00)\end{array}$ & \begin{tabular}{|c|}
0.0000 \\
$(0.00)$
\end{tabular} & $\begin{array}{c}0.0000 \\
(0.00)\end{array}$ & $\begin{array}{c}0.0000 \\
(0.00)\end{array}$ & $\begin{array}{c}0.0000 \\
(0.00)\end{array}$ & $\begin{array}{c}0.0000 \\
(0.00)\end{array}$ & $\begin{array}{c}0.0000 \\
(0.00)\end{array}$ & $\begin{array}{c}0.0000 \\
(0.00)\end{array}$ & $\begin{array}{c}0.0000 \\
(0.00)\end{array}$ & $\begin{array}{l}0.0000 \\
(0.00)\end{array}$ & 4768.17 \\
\hline Argentina & $\begin{array}{c}0.0000 \\
(0.00)\end{array}$ & $\begin{array}{c}0.0000 \\
(0.00)\end{array}$ & $\begin{array}{c}0.0000 \\
(0.00)\end{array}$ & $\begin{array}{c}0.0000 \\
(0.00)\end{array}$ & $\begin{array}{c}0.0000 \\
(0.00)\end{array}$ & $\begin{array}{c}0.0000 \\
(0.00)\end{array}$ & $\begin{array}{c}0.0000 \\
(0.00)\end{array}$ & $\begin{array}{c}0.0000 \\
(0.00)\end{array}$ & $\begin{array}{c}0.0000 \\
(0.00)\end{array}$ & $\begin{array}{c}0.0000 \\
(0.00)\end{array}$ & $\begin{array}{c}0.0000 \\
(0.00)\end{array}$ & $\begin{array}{c}0.3394 \\
(320.82)\end{array}$ & \begin{tabular}{|c|}
0.0000 \\
$(0.00)$
\end{tabular} & $\begin{array}{c}0.0000 \\
(0.00)\end{array}$ & $\begin{array}{c}0.0000 \\
(0.00)\end{array}$ & $\begin{array}{c}0.0000 \\
(0.00)\end{array}$ & $\begin{array}{c}0.0000 \\
(0.00)\end{array}$ & & & & & 945.22 \\
\hline Cameroon & $\begin{array}{c}0.0000 \\
(0.00)\end{array}$ & $\begin{array}{c}0.0000 \\
(0.00)\end{array}$ & $\begin{array}{c}0.3123 \\
(694.09)\end{array}$ & $\begin{array}{l}0.0000 \\
(0.00)\end{array}$ & $\begin{array}{l}0.0000 \\
(0.00)\end{array}$ & \begin{tabular}{|c|}
0.6877 \\
$(1528.69)$
\end{tabular} & $\begin{array}{c}0.0000 \\
(0.00)\end{array}$ & $\begin{array}{c}0.0000 \\
(0.00)\end{array}$ & $\begin{array}{c}0.0000 \\
(0.00)\end{array}$ & & & & \begin{tabular}{|c|}
0.0000 \\
$(0.00)$
\end{tabular} & $\begin{array}{c}0.0000 \\
(0.00)\end{array}$ & $\begin{array}{c}0.0000 \\
(0.00)\end{array}$ & & $\begin{array}{c}0.0000 \\
(0.00)\end{array}$ & & $\begin{array}{l}.0000 \\
0.00)\end{array}$ & $\begin{array}{l}0.00 \\
0.0\end{array}$ & & 2222.78 \\
\hline China & $\begin{array}{l}0.0000 \\
(0.00)\end{array}$ & $\begin{array}{l}0.0000 \\
(0.00)\end{array}$ & $\begin{array}{l}0.0000 \\
(0.00)\end{array}$ & $\begin{array}{l}0.0000 \\
(0.00)\end{array}$ & $\begin{array}{l}0.0000 \\
(0.00)\end{array}$ & $\begin{array}{l}0.0000 \\
(0.00)\end{array}$ & $\begin{array}{l}0.0000 \\
(0.00)\end{array}$ & $\begin{array}{l}0.0000 \\
(0.00)\end{array}$ & $\begin{array}{c}1.0000 \\
(1722.39)\end{array}$ & $\begin{array}{l}0.0000 \\
(0.00)\end{array}$ & & $\begin{array}{l}0.0000 \\
(0.00)\end{array}$ & \begin{tabular}{|l|}
0.0000 \\
$(0.00)$
\end{tabular} & $\begin{array}{l}0.0000 \\
(0.00)\end{array}$ & $\begin{array}{l}0.0000 \\
(0.00)\end{array}$ & $\begin{array}{l}0.0000 \\
(0.00)\end{array}$ & $\begin{array}{l}0.0000 \\
(0.00)\end{array}$ & & $\begin{array}{l}0.0000 \\
(0.00)\end{array}$ & $\begin{array}{l}0.0000 \\
(0.00)\end{array}$ & & 1722.39 \\
\hline Greece & $\begin{array}{l}0.0000 \\
(0.00)\end{array}$ & \begin{tabular}{|c|}
0.1823 \\
$(574.63)$ \\
\end{tabular} & $\begin{array}{l}0.0000 \\
(0.00)\end{array}$ & $\begin{array}{c}0.1917 \\
(604.00)\end{array}$ & $\begin{array}{l}0.0000 \\
(0.00)\end{array}$ & $\begin{array}{c}0.0901 \\
(284.03) \\
\end{array}$ & $\begin{array}{l}0.0000 \\
(0.00)\end{array}$ & $\begin{array}{c}0.3056 \\
(962.93)\end{array}$ & $\begin{array}{l}0.0000 \\
(0.00)\end{array}$ & $\begin{array}{c}0.0741 \\
(233.57)\end{array}$ & $\begin{array}{l}0.0000 \\
(0.00)\end{array}$ & $\begin{array}{l}0.0000 \\
(0.00)\end{array}$ & \begin{tabular}{|l|}
0.0000 \\
$(0.00)$
\end{tabular} & \begin{tabular}{|c|}
0.1291 \\
$(406.84)$
\end{tabular} & $\begin{array}{l}0.0000 \\
(0.00)\end{array}$ & $\begin{array}{l}0.0047 \\
(14.75)\end{array}$ & $\begin{array}{c}0.0000 \\
(0.00)\end{array}$ & $\begin{array}{l}0.0000 \\
(0.00)\end{array}$ & $\begin{array}{l}0.0000 \\
(0.00)\end{array}$ & $\begin{array}{l}0.0224 \\
(70.47)\end{array}$ & $\begin{array}{l}0.0000 \\
(0.00)\end{array}$ & 3151.22 \\
\hline Israel & $\begin{array}{c}0.0000 \\
(0.00)\end{array}$ & $\begin{array}{c}0.0000 \\
(0.00)\end{array}$ & $\begin{array}{c}0.0000 \\
(0.00)\end{array}$ & $\begin{array}{c}0.0000 \\
(0.00)\end{array}$ & $\begin{array}{c}0.7316 \\
(649.14)\end{array}$ & $\begin{array}{c}0.0000 \\
(0.00)\end{array}$ & $\begin{array}{l}0.0000 \\
(0.00)\end{array}$ & $\begin{array}{c}0.0000 \\
(0.00)\end{array}$ & $\begin{array}{c}0.0000 \\
(0.00)\end{array}$ & $\begin{array}{c}0.0000 \\
(0.00)\end{array}$ & $\begin{array}{l}0.0000 \\
(0.00)\end{array}$ & $\begin{array}{c}0.0000 \\
(0.00)\end{array}$ & \begin{tabular}{|c|}
0.0000 \\
$(0.00)$
\end{tabular} & $\begin{array}{c}0.0000 \\
(0.00)\end{array}$ & $\begin{array}{c}0.0000 \\
(0.00)\end{array}$ & $\begin{array}{c}0.0000 \\
(0.00)\end{array}$ & $\begin{array}{c}0.2684 \\
(238.19)\end{array}$ & $\begin{array}{c}0.0000 \\
(0.00)\end{array}$ & $\begin{array}{c}0.0000 \\
(0.00)\end{array}$ & $\begin{array}{c}0.0000 \\
(0.00)\end{array}$ & $\begin{array}{c}0.0000 \\
(0.00)\end{array}$ & 887.33 \\
\hline Malaysia & $\begin{array}{c}0.3799 \\
(392.26)\end{array}$ & \begin{tabular}{|c|}
0.6201 \\
$(640.24)$
\end{tabular} & $\begin{array}{l}0.0000 \\
(0.00)\end{array}$ & $\begin{array}{l}0.0000 \\
(0.00)\end{array}$ & $\begin{array}{l}0.0000 \\
(0.00)\end{array}$ & $\begin{array}{l}0.0000 \\
(0.00)\end{array}$ & $\begin{array}{l}0.0000 \\
(0.00)\end{array}$ & $\begin{array}{l}0.0000 \\
(0.00)\end{array}$ & $\begin{array}{c}0.0000 \\
(0.00)\end{array}$ & $\begin{array}{l}0.0000 \\
(0.00)\end{array}$ & $\begin{array}{l}0.0000 \\
(0.00)\end{array}$ & $\begin{array}{l}0.0000 \\
(0.00)\end{array}$ & \begin{tabular}{|l|}
0.0000 \\
$(0.00)$
\end{tabular} & $\begin{array}{l}0.0000 \\
(0.00)\end{array}$ & $\begin{array}{l}0.0000 \\
(0.00)\end{array}$ & $\begin{array}{l}0.0000 \\
(0.00)\end{array}$ & $\begin{array}{c}0.0000 \\
(0.00)\end{array}$ & $\begin{array}{l}0.0000 \\
(0.00)\end{array}$ & $\begin{array}{l}0.0000 \\
(0.00)\end{array}$ & $\begin{array}{l}0.0000 \\
(0.00)\end{array}$ & $\begin{array}{l}0.0000 \\
(0.00)\end{array}$ & 1032.50 \\
\hline Nigeria & $\begin{array}{l}0.0000 \\
(0.00)\end{array}$ & $\begin{array}{c}0.0000 \\
(0.00)\end{array}$ & $\begin{array}{l}0.0000 \\
(0.00)\end{array}$ & $\begin{array}{l}0.0000 \\
(0.00)\end{array}$ & $\begin{array}{l}0.0000 \\
(0.00)\end{array}$ & $\begin{array}{l}0.0000 \\
(0.00)\end{array}$ & $\begin{array}{l}0.0000 \\
(0.00)\end{array}$ & $\begin{array}{l}0.0000 \\
(0.00)\end{array}$ & \begin{tabular}{|c|}
0.8325 \\
$(1062.64)$
\end{tabular} & $\begin{array}{l}0.0000 \\
(0.00)\end{array}$ & $\begin{array}{l}0.0000 \\
(0.00)\end{array}$ & $\begin{array}{c}0.1675 \\
(213.80)\end{array}$ & \begin{tabular}{|c|}
0.0000 \\
$(0.00)$
\end{tabular} & $\begin{array}{l}0.0000 \\
(0.00)\end{array}$ & $\begin{array}{l}0.0000 \\
(0.00)\end{array}$ & $\begin{array}{l}0.0000 \\
(0.00)\end{array}$ & $\begin{array}{c}0.0000 \\
(0.00)\end{array}$ & $\begin{array}{l}0.0000 \\
(0.00)\end{array}$ & $\begin{array}{l}0.0000 \\
(0.00)\end{array}$ & $\begin{array}{l}0.0000 \\
(0.00)\end{array}$ & $\begin{array}{l}0.0000 \\
(0.00)\end{array}$ & 1276.44 \\
\hline \begin{tabular}{|l|} 
Russian \\
Federation \\
\end{tabular} & $\begin{array}{c}0.0000 \\
(0.00)\end{array}$ & \begin{tabular}{|c|}
0.1477 \\
$(237.78)$ \\
\end{tabular} & $\begin{array}{l}0.0000 \\
(0.00)\end{array}$ & $\begin{array}{l}0.0600 \\
(96.63) \\
\end{array}$ & $\begin{array}{c}0.0000 \\
(0.00)\end{array}$ & $\begin{array}{l}0.0000 \\
(0.00)\end{array}$ & $\begin{array}{l}.0000 \\
(0.00)\end{array}$ & $\begin{array}{c}0.0000 \\
(0.00)\end{array}$ & $\begin{array}{c}0.0000 \\
(0.00) \\
\end{array}$ & $\begin{array}{l}0.0560 \\
(90.20) \\
\end{array}$ & $\begin{array}{l}0.0000 \\
(0.00)\end{array}$ & $\begin{array}{c}0.0000 \\
(0.00)\end{array}$ & \begin{tabular}{|c|}
0.0000 \\
$(0.00)$ \\
\end{tabular} & $\begin{array}{c}0.0000 \\
(0.00)\end{array}$ & $\begin{array}{l}0.0000 \\
(0.00)\end{array}$ & $\begin{array}{c}0.1263 \\
(203.35) \\
\end{array}$ & $\begin{array}{c}0.0000 \\
(0.00)\end{array}$ & $\begin{array}{c}.3028 \\
(487.57) \\
\end{array}$ & $\begin{array}{l}0.0183 \\
(29.49) \\
\end{array}$ & $\begin{array}{l}0.0000 \\
(0.00)\end{array}$ & $\begin{array}{c}0.2890 \\
(465.37) \\
\end{array}$ & 1610.39 \\
\hline \begin{tabular}{|l|} 
South \\
Africa
\end{tabular} & $\begin{array}{c}0.7685 \\
(1602.83)\end{array}$ & $\begin{array}{c}0.0000 \\
(0.00)\end{array}$ & $\begin{array}{c}0.0000 \\
(0.00)\end{array}$ & $\begin{array}{l}0.0000 \\
(0.00)\end{array}$ & $\begin{array}{c}0.0000 \\
(0.00)\end{array}$ & $\begin{array}{l}0.0000 \\
(0.00)\end{array}$ & $\begin{array}{c}0.0000 \\
(0.00)\end{array}$ & $\begin{array}{c}0.0000 \\
(0.00)\end{array}$ & $\begin{array}{c}0.0000 \\
(0.00)\end{array}$ & $\begin{array}{c}0.0000 \\
(0.00)\end{array}$ & $\begin{array}{c}0.0000 \\
(0.00)\end{array}$ & $\begin{array}{c}0.0000 \\
(0.00)\end{array}$ & \begin{tabular}{|c|}
0.0000 \\
$(0.00)$
\end{tabular} & $\begin{array}{c}0.0000 \\
(0.00)\end{array}$ & $\begin{array}{c}0.0000 \\
(0.00)\end{array}$ & \begin{tabular}{|c|}
0.0000 \\
$(0.00)$
\end{tabular} & $\begin{array}{c}0.0000 \\
(0.00)\end{array}$ & $\begin{array}{l}0.0000 \\
(0.00)\end{array}$ & $\begin{array}{c}0.2315 \\
(482.78)\end{array}$ & $\begin{array}{c}0.0000 \\
(0.00)\end{array}$ & $\begin{array}{l}0.0000 \\
(0.00)\end{array}$ & 2085.61 \\
\hline Switzerland & $\begin{array}{c}0.0000 \\
(0.00)\end{array}$ & $\begin{array}{c}0.0000 \\
(0.00)\end{array}$ & $\begin{array}{c}0.0000 \\
(0.00)\end{array}$ & $\begin{array}{c}0.0000 \\
(0.00)\end{array}$ & $\begin{array}{c}0.0000 \\
(0.00)\end{array}$ & $\begin{array}{c}0.0000 \\
(0.00)\end{array}$ & \begin{tabular}{|c|}
0.4261 \\
$(342.07)$
\end{tabular} & $\begin{array}{c}0.0000 \\
(0.00)\end{array}$ & $\begin{array}{c}0.0000 \\
(0.00)\end{array}$ & $\begin{array}{c}0.0000 \\
(0.00)\end{array}$ & $\begin{array}{c}0.0000 \\
(0.00)\end{array}$ & $\begin{array}{c}0.0000 \\
(0.00)\end{array}$ & $\begin{array}{c}0.0000 \\
(0.00)\end{array}$ & $\begin{array}{c}0.0000 \\
(0.00)\end{array}$ & $\begin{array}{c}0.0000 \\
(0.00)\end{array}$ & $\begin{array}{c}0.0000 \\
(0.00)\end{array}$ & $\begin{array}{c}0.0000 \\
(0.00)\end{array}$ & $\begin{array}{c}0.0000 \\
(0.00)\end{array}$ & $\begin{array}{c}0.1655 \\
(132.84)\end{array}$ & $\begin{array}{c}0.0000 \\
(0.00)\end{array}$ & \begin{tabular}{|c|}
0.4084 \\
$(327.86)$
\end{tabular} & 802.78 \\
\hline Others & $\begin{array}{c}0.0902 \\
(1929.23) \\
\end{array}$ & \begin{tabular}{|c|}
0.0872 \\
$(1866.02)$ \\
\end{tabular} & $\begin{array}{c}0.0648 \\
(1385.80) \\
\end{array}$ & $\begin{array}{c}0.0596 \\
(1274.89) \\
\end{array}$ & $\begin{array}{c}0.0279 \\
(597.79) \\
\end{array}$ & $\begin{array}{l}0.0038 \\
(81.99) \\
\end{array}$ & \begin{tabular}{|l|}
0.0045 \\
$(96.44)$ \\
\end{tabular} & $\begin{array}{c}0.0000 \\
(0.00)\end{array}$ & \begin{tabular}{|c|}
0.3164 \\
$(6769.38)$ \\
\end{tabular} & $\begin{array}{c}0.1629 \\
(3486.11) \\
\end{array}$ & $\begin{array}{c}0.0000 \\
(0.00)\end{array}$ & $\begin{array}{r}0.0189 \\
(405.25) \\
\end{array}$ & \begin{tabular}{|c|}
0.0172 \\
$(368.76)$ \\
\end{tabular} & $\begin{array}{c}0.0000 \\
(0.00) \\
\end{array}$ & $\begin{array}{c}0.0000 \\
(0.00)\end{array}$ & \begin{tabular}{|c|}
0.0109 \\
$(233.35)$ \\
\end{tabular} & $\begin{array}{c}0.0000 \\
(0.00)\end{array}$ & $\begin{array}{c}0.0000 \\
(0.00) \\
\end{array}$ & $\begin{array}{c}0.0132 \\
(282.91) \\
\end{array}$ & $\begin{array}{c}0.0000 \\
(0.00)\end{array}$ & \begin{tabular}{|c|}
$\mathbf{0 . 1 2 2 3}$ \\
$(\mathbf{2 6 1 6 . 7 5 )}$ \\
\end{tabular} & 21394.67 \\
\hline \begin{tabular}{|l|} 
Total \\
Import
\end{tabular} & 8304.75 & 5363.69 & 3354.29 & 3325.03 & 21134.90 & 3814.59 & 4351.50 & 5091.52 & 41676.77 & 4224.93 & 141.25 & 2133.71 & 869.79 & 2002.86 & 1443.53 & 458.60 & 1285.09 & 1126.28 & 1562.94 & 318.67 & 19314.03 & \\
\hline Percentage & 101.52 & 88.76 & 75.55 & 65.05 & 128.81 & 86.43 & 125.58 & 85.49 & 117.79 & 88.61 & 14.94 & 95.99 & 50.50 & 63.56 & 162.68 & 44.42 & 100.68 & 69.94 & 74.94 & 39.70 & 90.27 & \\
\hline $\mid \begin{array}{l}\% \\
\text { Increase/ } \\
\text { Decrease }\end{array}$ & 1.52 & -11.24 & -24.45 & -34.95 & 28.81 & -13.57 & 25.58 & -14.51 & 17.79 & -11.39 & -85.06 & -4.01 & -49.50 & -36.44 & 62.68 & -55.58 & 0.68 & -30.06 & -25.06 & -60.30 & -9.73 & \\
\hline
\end{tabular}

Note: Figures in the parenthesis indicate average quantity of import (in tons) by India 
The transitional probability matrices are presented in Table 5 and 6 . The results indicated the changes in the direction of trade of Indian cotton imports in both periods which helped in describing the changing direction during the post-WTO period over the pre-WTO period.

It is observed from Table 5 that during preWTO period all the countries except Pakistan, Sudan and USA were highly unstable exporter of cotton to India and could not retain the previous year's share of imports. Cent per cent of imports of previous year was diverted.

The sum of diversified and retained import from each country was given below in the form of transitional probabilities. The picture of import indicates that there was 233.75 per cent increase in import from Russian Federation followed by 233.38 per cent from Malaysia, 177.17 per cent from Cameroon, 104.62 per cent from others and 97.71 per cent from Cote d'lvoire during pre-WTO period. There was 100 per cent reduction in import from Egypt, Uzbekistan and Austria followed by 93.34 per cent from Mali, 61.04 per cent reduction in import from Burkina Faso, 58.73 per cent from Tanzania and 28.13 per cent reduction in import from Pakistan during the pre-WTO period.

The trade pattern and direction of cotton imports during post-WTO period is given in the Table 6. It is observed from Table 6 that during post-WTO period Benin, Cote d'lvoire, Sudan, Uzbekistan, Argentina, Cameroon, China, Israel, Malaysia, Nigeria and Switzerland were highly unstable exporter of cotton to India and could not retain the previous year's share of imports. USA, Russian Federation, Mali, Egypt and South Africa were the stable exporters of cotton to India during post-WTO period. The sum of diversified and retained import from each country was given below the transitional probabilities. The picture of import indicates that there was 62.68 per cent increase in import from Israel followed by 28.81 per cent from Egypt, 25.58 per cent from Sudan, 17.79 per cent from USA and 1.52 per cent from Australia during post-WTO period. There was 85.06 per cent reduction in import from Argentina followed by 60.30 per cent from Switzerland, 55.58 per cent reduction in import from Malaysia, 49.50 per cent from China and 36.44 per cent reduction in import from Greece during the post-WTO period.

\section{Export competitiveness of Indian cotton}

As per the theory of comparative advantage, a particular nation can enhance the resource use efficiency and there by the net welfare by producing and exporting commodities in which it has greater comparative advantage than others, so as to maximize its exports revenue.

The Nominal Protection Coefficient (NPC) technique explains the comparative advantage enjoyed by the commodity in the context of free trade. The export competitiveness of cotton was analyzed by using nominal protection coefficient and the values of NPC for Indian cotton are presented in Table 7. The results of the NPC values for both the pre-WTO and post-WTO period indicated that the coefficients were less than one for all the years. It indicate that there was a more scope for export of cotton i.e. cotton was disprotected in India. The average NPC value for pre-WTO period (0.34) and post-WTO period (0.38) indicated that the unit price of the Indian cotton in the domestic market was not much competitive in the international market. The value of NPC was noticed highest (0.48) in 1987-88 during pre-WTO period and (0.50) in 2008-09 during post -WTO period. The lowest value of 0.19 was observed during 1990-91 in pre-WTO period and 0.20 during 
1996-97 in post-WTO period. China, Turkey, Indonesia, Japan and Thailand were the stable markets of Indian cotton during pre-WTO period whereas during the post-WTO period China, Japan and Malaysia were the stable markets of Indian cotton. During the preWTO period none of the country was stable exporter of cotton to India whereas during post-WTO period USA and Russian Federation were the stable exporters of cotton to India. An average NPC values for the domestic market price was 0.34 and 0.38 during pre- and post-WTO periods, respectively indicated more competitiveness of Indian cotton in the international market.

\section{References}

Guledgudda, S.S., 2005. Production and Export Performance of Cashew - An Economic Analysis. Ph.D. Thesis, Uni. of Agric. Sci., Dharwad.
Jayesh, T., 2001. Production and Export Performance of Selected Spices in South India: An Economic Analysis, $M$. Sc. (Agri.) Thesis, Uni. of Agric. Sci., Dharwad.

Mahesh, N., 2009. Economic Constraints Facing the Indian Tea Industry: Strategies for Post - WTO era, Ph. D. Thesis, Univ. Agric. Sci., Bangalore, Karnataka, India.

Pramod Kumar et al., 2007. Changing direction of Indian mango exports. Ind. J. Agric. Mktg., 21(1): 130-137.

Reza Sedaghat et al., 2008. Study of Structural Changes in Trade Directions of Pistachio from Iran, Mysore J. Agric, Sci., 42 (3): 510-514.

Sadavatti, P.M., 2006. Exports of Basmati Rice and its stability: Markov Chain Analysis, Ind. J. Agril. Mktg., 20 (1): 11-16.

\section{How to cite this article:}

Lamtule, J.A., P.P. Sawant and Deshmukh, R.G. 2018. Direction and Competitiveness of Cotton Export under WTO Regime. Int.J.Curr.Microbiol.App.Sci. 7(01): 834-844.

doi: https://doi.org/10.20546/ijcmas.2018.701.102 